ARTICLE

\title{
Engineered niches support the development of human dendritic cells in humanized mice
}

\author{
Giorgio Anselmi ${ }^{1,2,7}$, Kristine Vaivode ${ }^{1,2}$, Charles-Antoine Dutertre ${ }^{3}$, Pierre Bourdely ${ }^{1,2}$, \\ Yoann Missolo-Koussou (10 4 ${ }^{4}$, Evan Newell ${ }^{3}$, Oliver Hickman ${ }^{1,2,8}$, Kristie Wood ${ }^{5,9}$, Alka Saxena ${ }^{5}$, Julie Helft (1) ${ }^{4}$, \\ Florent Ginhoux (i) ${ }^{3} \&$ Pierre Guermonprez ${ }^{1,2,6 凶}$
}

Classical dendritic cells (cDCs) are rare sentinel cells specialized in the regulation of adaptive immunity. Modeling CDC development is crucial to study CDCs and harness their therapeutic potential. Here we address whether CDCs could differentiate in response to trophic cues delivered by mesenchymal components of the hematopoietic niche. We find that mesenchymal stromal cells engineered to express membrane-bound FLT3L and stem cell factor (SCF) together with CXCL12 induce the specification of human CDCs from CD34+ hematopoietic stem and progenitor cells (HSPCs). Engraftment of engineered mesenchymal stromal cells (eMSCs) together with CD34+ HSPCs creates an in vivo synthetic niche in the dermis of immunodeficient mice driving the differentiation of $\mathrm{CDCs}$ and $\mathrm{CD}_{123^{+}} \mathrm{AXL}^{+}$ CD327+ pre/AS-DCs. cDC2s generated in vivo display higher levels of resemblance with human blood cDCs unattained by in vitro-generated subsets. Altogether, eMSCs provide a unique platform recapitulating the full spectrum of $C D C$ subsets enabling their functional characterization in vivo.

\footnotetext{
${ }^{1}$ Centre for Inflammation Biology and Cancer Immunology, The Peter Gorer Department of Immmunobiology, King's College London, London, UK. ${ }^{2}$ Cancer Research UK, King's Health Partners Cancer Centre, King's College London, London, UK. ${ }^{3}$ Singapore Immunology Network (SlgN), A*STAR, Singapore, Singapore. ${ }^{4}$ Paris-Sciences-Lettres University, Institut Curie Research Center, INSERM U932 \& SiRIC, Translational Immunotherapy Team, Paris, France. ${ }^{5}$ National Institute of Health Research Biomedical Research Centre at Guy's and St Thomas' Hospital and King's College London, London, UK. ${ }^{6}$ Université de Paris, Centre for Inflammation Research, CNRS ERL8252, INSERM1149, Paris, France. ${ }^{7}$ Present address: MRC Molecular Hematology Unit, MRC Weatherall Institute of Molecular Medicine, Radcliffe Department of Medicine, University of Oxford, Oxford, UK. ${ }^{8}$ Present address: Drug Target Discovery Team, Division of Breast Cancer Research, Institute of Cancer Research, London, UK. ${ }^{9}$ Present address: Labcyte Ltd, Norton Canes, Cannock, Staffordshire, UK. ${ }^{凶}$ email: pierre.guermonprez@kcl.ac.uk
} 
C lassical human dendritic cells (cDCs) are sentinels of the immune system with a unique ability to regulate the function of T lymphocytes ${ }^{1}$. Dendritic cells (DCs) can induce immune tolerance ${ }^{2}$ or drive the development of immunity ${ }^{3}$.

The analysis of blood circulating subsets has revealed that $\mathrm{CDCs}$ consist in two major subtypes: $\mathrm{CD} 141^{+} \mathrm{XCR} 1^{+} \mathrm{Clec} 9 \mathrm{~A}^{+} \mathrm{DCs}$ $(\mathrm{cDC} 1)$ and $\mathrm{CD}_{1} \mathrm{c}^{+} \mathrm{CD} 11 \mathrm{c}^{+} \mathrm{CD} 172 \mathrm{a}(\mathrm{SIRPa})^{+} \mathrm{DCs}(\mathrm{cDC} 2 \mathrm{~s})^{4-6}$. Both $\mathrm{cDC} 1 \mathrm{~s}$ and $\mathrm{cDC} 2 \mathrm{~s}$ arise from a bone marrow committed progenitor $^{7}$ or from early IRF8 ${ }^{+}$multipotent progenitors ${ }^{8,9}$, which generate a common circulating precursor ${ }^{10}$ that progressively diverge in pre-cDC1s and pre-cDC2s ${ }^{10-12}$. Type 1 DCs are conserved between mouse and human, and they share the expression of specific surface markers such as $\mathrm{Clec} 9 \mathrm{~A}^{13}$ and $\mathrm{XCR} 1^{5}$, as well as the transcription factor (TF) IRF8, which is essential for the development of murine $\mathrm{cDC} 1 \mathrm{~s}^{4-6,13-15}$. Conversely, human $\mathrm{CD} 1 \mathrm{c}^{+}$type 2 DCs have been shown to express the IRF4 $\mathrm{TF}^{16}$, which controls the development of their phenotypic equivalent in the mouse model ${ }^{16,17}$. This rather simple picture is complicated by the diversity of $\mathrm{CD}^{+} \mathrm{c}^{+}$cells, which encompass migratory DCs and CD14 int inflammatory DCs recruited during inflammation ${ }^{18,19}$. More recently, unbiased approaches have uncovered a deeper complexity in the DC network with the identification of two types of $\mathrm{cDC} 2 \mathrm{~s}$ with distinct transcriptional profiles and the identification of $\mathrm{AXL}^{+} \mathrm{CD} 11 \mathrm{c}^{+} \mathrm{CD} 1 \mathrm{c}^{+}$cells, which have been proposed to act as a precursor for $\mathrm{CDCs}^{12,20}$.

Human hematopoietic progenitors reside in the stem cells niche of the bone marrow. Genetic studies in the murine model identified three essential factors supporting HSPCs homeostasis: the membrane-bound form of stem cell factor (SCF/KITL $)^{21,22}$, the $\mathrm{C}-\mathrm{X}-\mathrm{C}$ motive chemokine 12 (CXCL12) $^{23,24}$ and thrombopoietin $(\mathrm{TPO})^{25,26}$. In the bone marrow, perivascular mesenchymal stromal cells have been described as the main source of SCF and other niche factors ${ }^{27}$. At steady state, Flt3-ligand (FLT3L) is delivered as a membrane-bound precursor expressed on radioresistant stromal cells ${ }^{28-30}$. After egressing from the bone marrow, DC precursors circulate in the blood and seed the peripheral tissues $^{31}$. In the lymph node, stromal fibroblastic reticular cells provide FLT3L ${ }^{32}$ and FLT3-dependent proliferation of $\mathrm{CDC}$ in periphery is required for their maintenance ${ }^{33,34}$.

Modeling the development of cDCs in culture systems is instrumental to better understand their ontogeny and define their immunological function. Pioneer work from Banchereau et al. ${ }^{35}$ have identified that granulocyte-macrophage colony-stimulating factor (GM-CSF) and tumor necrosis factor- $\alpha$ (TNF- $\alpha$ ) cooperate to produce $\mathrm{CD} \mathrm{a}^{+}$cells with features of Langerhans cells from CD $34^{+}$hematopoietic stem and progenitor cells (HSPCs). Sallusto et al. ${ }^{36}$ have shown that GM-CSF and interleukin (IL)-4 induce the differentiation of $\mathrm{CD} \mathrm{c}^{+} \mathrm{CD} 1 \mathrm{a}^{+}$inflammatory DCs from CD14 monocytes. More recent work has demonstrated that FLT3L (with TPO or with SCF/KITL, GM-CSF, and IL-4) is instrumental in generating $\mathrm{CD} 141^{+} \mathrm{cDC} 1$ s aligning phenotypically and functionally with $\mathrm{cDC} 1 \mathrm{~s}^{7,8,37-39}$. This is in line with the crucial role of FLT3L, engaging the Flt3 receptor tyrosine kinase $^{40,41}$ in controlling DC homeostasis both in mice $31,33,42,43$ and humans ${ }^{10,28,44,45}$. Moreover, the activation of Notch signaling pathway has been shown to further improve the in vitro differentiation of both human and mouse $\mathrm{cDC} 1 \mathrm{~s}^{46,47}$. Despite the successes in modeling $\mathrm{CDC} 1$ differentiation in vitro, $\mathrm{CD}_{1} \mathrm{c}^{+}$cells found in culture of $\mathrm{CD} 34^{+}$HSPCs either align poorly with bona fide blood circulating $\mathrm{cDC} 2 \mathrm{~s}^{38}$ or were not extensively characterized 46,47 .

Recapitulating human cDC development in vivo has the potential to greatly improve our understanding of DC biology and facilitate its translational applications. Human cDCs have been found in stably reconstituted humanized mice treated with supraphysiological concentration of human FLT3L $28,48,49$.
However, the generated $\mathrm{CD} 11 \mathrm{c}^{+} \mathrm{CD} 141^{+}$and $\mathrm{CD} 11 \mathrm{c}^{+} \mathrm{CD} 1 \mathrm{c}^{+}$ cells were poorly characterized and their dissemination to peripheral tissues has rarely been assessed ${ }^{50}$.

Here we aimed at modeling human $\mathrm{cDC}$ development by providing physiological factors associated to hematopoietic niches. We found that engineered mesenchymal stromal cells (eMSCs) expressing a combination of membrane-bound FLT3L and SCF/KITL together with CXCL12 provide a scaffold for human $\mathrm{CDC}$ differentiation. Engraftment of eMSCs along with CD34 ${ }^{+}$HSPCs leads to the local development of $\mathrm{cDCs}$ in immunodeficient mice. This in vivo system recapitulates the differentiation of not only pDCs, $\mathrm{cDC} 1 \mathrm{~s}$, and $\mathrm{cDC} 2 \mathrm{~s}$ but also pre/ AS-DCs, and reaches an unmatched level of similarity of the generated $\mathrm{cDC} 2 \mathrm{~s}$ with their blood counterparts.

\section{Results}

Transmembrane FLT3L drives human DC differentiation in vitro. We hypothesized that the interaction of hematopoietic progenitors with membrane-bound factors expressed by stromal cells of the niche would promote the specification of the $\mathrm{CDC}$ lineage.

To test this, we engineered a bone marrow-derived murine mesenchymal cell line (MS5) 7,51 to stably and homogeneously express the transmembrane form of human FLT3L (MS5_F) as probed by flow cytometry (Fig. 1a). Co-culture of MS5_F with $\mathrm{CD}_{4}{ }^{+} \mathrm{HSPCs}$ drives the appearance of $\mathrm{cDC1}$-like Clec9A ${ }^{+}$ $\mathrm{CD}_{141}{ }^{+}$and cDC2-like CD14-CD1c ${ }^{+}$cells. Importantly, MS5_F is more efficient than recombinant soluble FLT3L (MS5 + recFL) in generating cDC-like cells (Fig. 1b).

In contrast, OP9 ${ }^{52}$ hematogenic stromal cells stably expressing membrane-bound FLT3L (OP9_F) were less efficient than MS5_F in driving cDC differentiation (Fig. 1c). Besides, MS5_F also promoted the appearance of $\mathrm{CD} 123^{+} \mathrm{CD} 303 / 4^{+}$cells resembling either pDCs or pre/AS-DCs ${ }^{12,20}$ (Supplementary Fig. 1a, b).

Next, we wanted to test whether cell-to-cell interactions mediate the differentiation of cDCs driven by FLT3L-expressing MS5 stromal cells. Using transwell permeable to soluble factors but preventing cognate interactions, we found that direct contact is required to support efficiently $\mathrm{cDC}$ differentiation (Fig. 1d).

Collectively, these data show that membrane FLT3L expression in stromal cells provide an improved platform to trigger the differentiation of $\mathrm{cDC}$-like cells from $\mathrm{CD} 34^{+}$HSPCs in vitro via cell-to-cell contact.

CXCL12 and SCF improve FLT3L-driven DC differentiation. Next, we sought to improve the efficiency of $\mathrm{cDC}$ production in MS5_F by co-expressing additional niche factors. We focused on SCF, CXCL12, and TPO because of their essential role in supporting HSPCs maintenance in the bone marrow niche $21,23-26,53$. SCF had also been extensively used in previously published DC culture protocols $7,38,39,54$.

To this end, we generated a collection of MS5 stromal cells stably expressing either one, two, three, or four human factors by combining CXCL12, TPO, and membrane-bound SCF/KITL, with or without membrane-bound FLT3L (Supplementary Fig. 2a).

We screened this collection of eMSC lines based on their ability to support human $\mathrm{cDC}$ differentiation from cord blood-derived CD34 ${ }^{+}$HSPCs.

At day 15, only FLT3L-expressing eMSCs successfully supported the differentiation of $\mathrm{CD}_{141}{ }^{+} \mathrm{Clec} 9 \mathrm{~A}^{+}$and $\mathrm{CD} 14^{-}$ CD $1 c^{+}$cells (Fig. 2a and Supplementary Figs. 2b and 3a). We conclude that FLT3L is necessary for the differentiation of $\mathrm{cDCs}$ using eMSCs. Besides, optimal cDC production was obtained in 
a

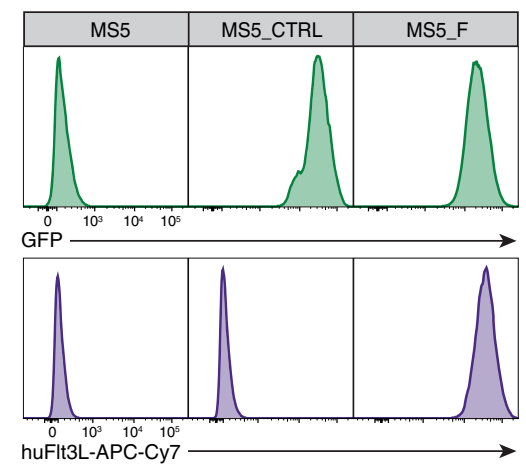

b
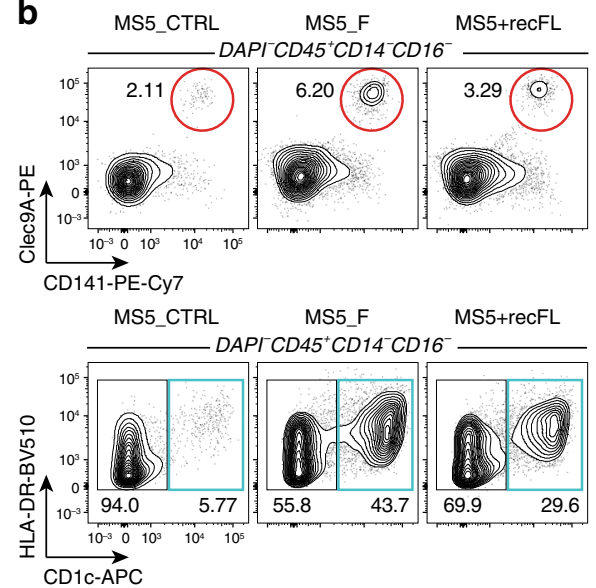
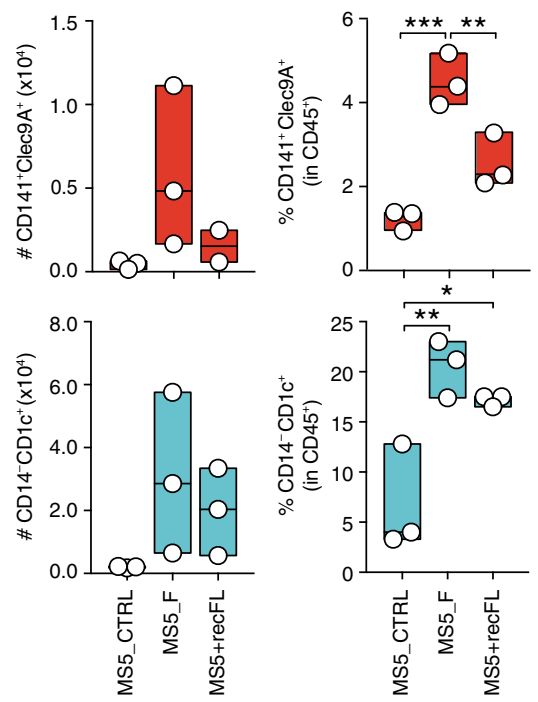

C
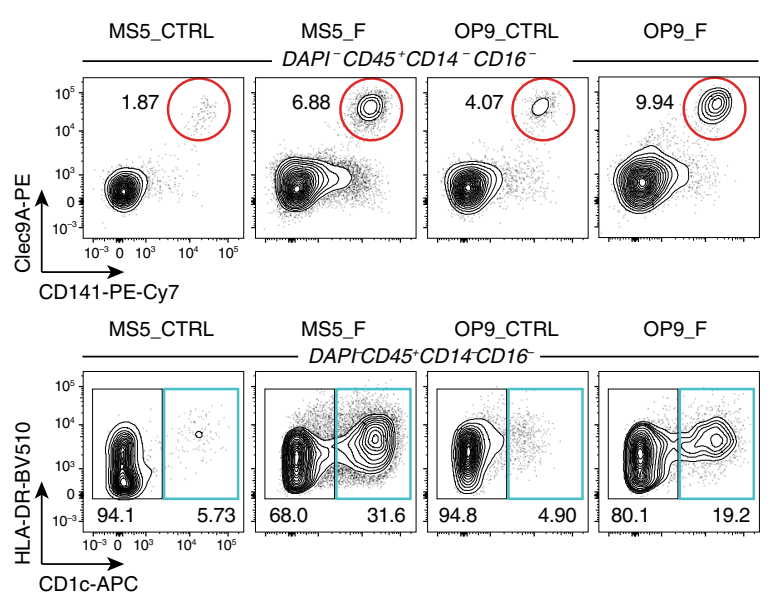

MS5F OP9 CTRL

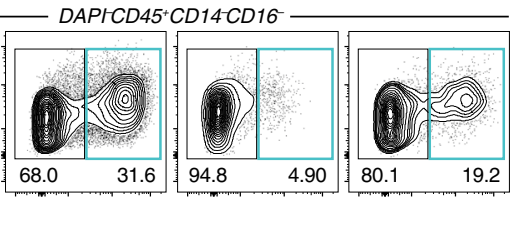

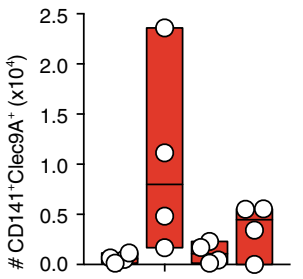

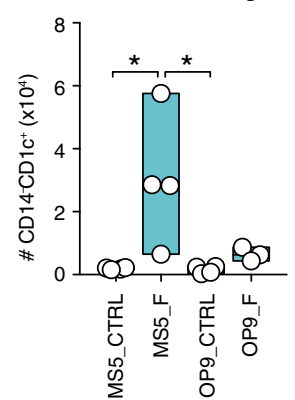

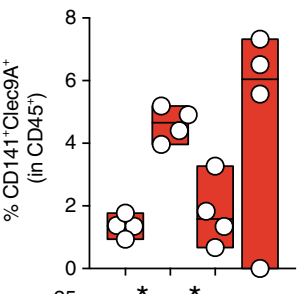

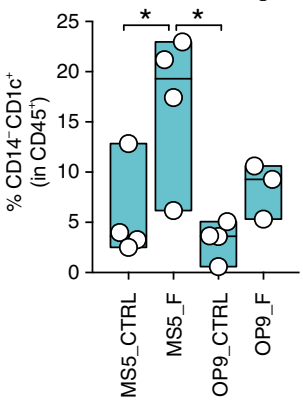

d

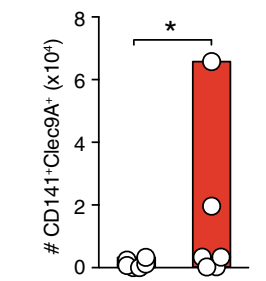

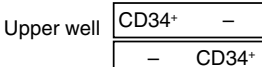

Lower well $\begin{array}{cc}- & \text { CD34+ } \\ \text { MS5_F } & \text { MS5_F }\end{array}$

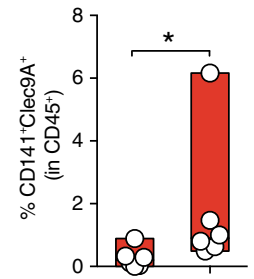

Upper well $\mathrm{CD} 34^{+}$ Lower well \begin{tabular}{ccc}
- & CD34 \\
MS5_F & MS5_F \\
\hline
\end{tabular}

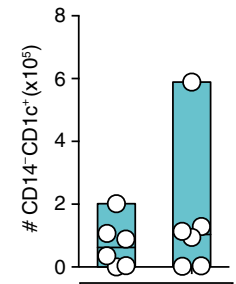

Upper we

Lower well \begin{tabular}{rr}
- & CD34 \\
MS5_F & MS5_F \\
\hline
\end{tabular}

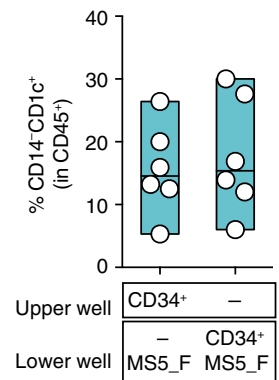

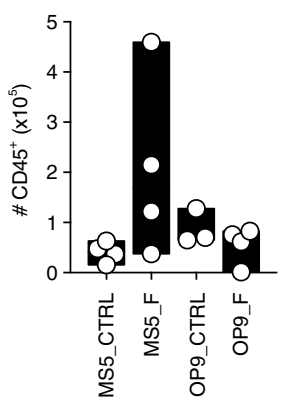

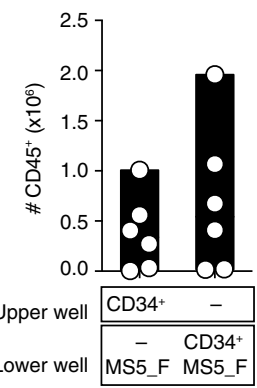

Fig. 1 Transmembrane FLT3L drives human DC differentiation in vitro. a Expression of membrane-bound FLT3L in mouse bone marrow-derived stromal cells engineered to express human FLT3L (MS5_F) and control (MS5_CTRL). b Human CDC subsets differentiated in vitro from CD34 ${ }^{+}$cord blood-derived HSPCs cultured with MS5 expressing membrane-bound FLT3L (MS5_F) or MS5 supplemented with recombinant human FLT3L (MS5+recFL) at day 15 $\left(n=3\right.$ donors in one experiment). ${ }^{\star} p<0.05,{ }^{\star \star} p<0.01,{ }^{\star \star \star} p<0.001$, one-way ANOVA test with Tukey's multiple comparisons. c Representative flow cytometry plots and quantification of human $\mathrm{CDC}$ subsets differentiated in vitro from cord blood-derived CD34+ progenitors in culture with mouse stromal cell lines MS5 and OP9 expressing human FLT3L (MS5_F and OP9_F) at day 15 ( $n=4$ donors in one experiment). ${ }^{*} p<0.05$, one-way ANOVA test with Tukey's multiple comparisons). d Absolute number and frequency of CD141+Clec9A ${ }^{+}$and $\mathrm{CD} 14^{-} \mathrm{CD} 1 \mathrm{c}^{+}$human $\mathrm{CDCs}$ differentiated from CD34+ $\mathrm{HSPCs}$ in direct contact (lower well) or physically separated (upper well) from engineered MS5_F. DC differentiation was assessed at day 15 by flow cytometry ( $n=$ 6 donors in three independent experiments). ${ }^{*} p<0.05$, two-tailed paired Student's $t$-test. Data are presented as floating bars ranging from min to max and line represents median (b-d). 
a

\begin{tabular}{r|c|c|c|c|c|c|c|c|c|} 
FIt3L & - & + & + & + & + & + & + & + & + \\
\hline SCF & - & - & + & - & + & - & + & - & + \\
\hline TPO & - & - & - & - & - & + & + & + & + \\
\hline \multirow{2}{*}{ CXCL12 } & - & - & - & + & + & - & - & + & + \\
\cline { 2 - 11 } & CTRL & F & FS & F12 & FS12 & FT & FST & FT12 & FST12 \\
\hline
\end{tabular}
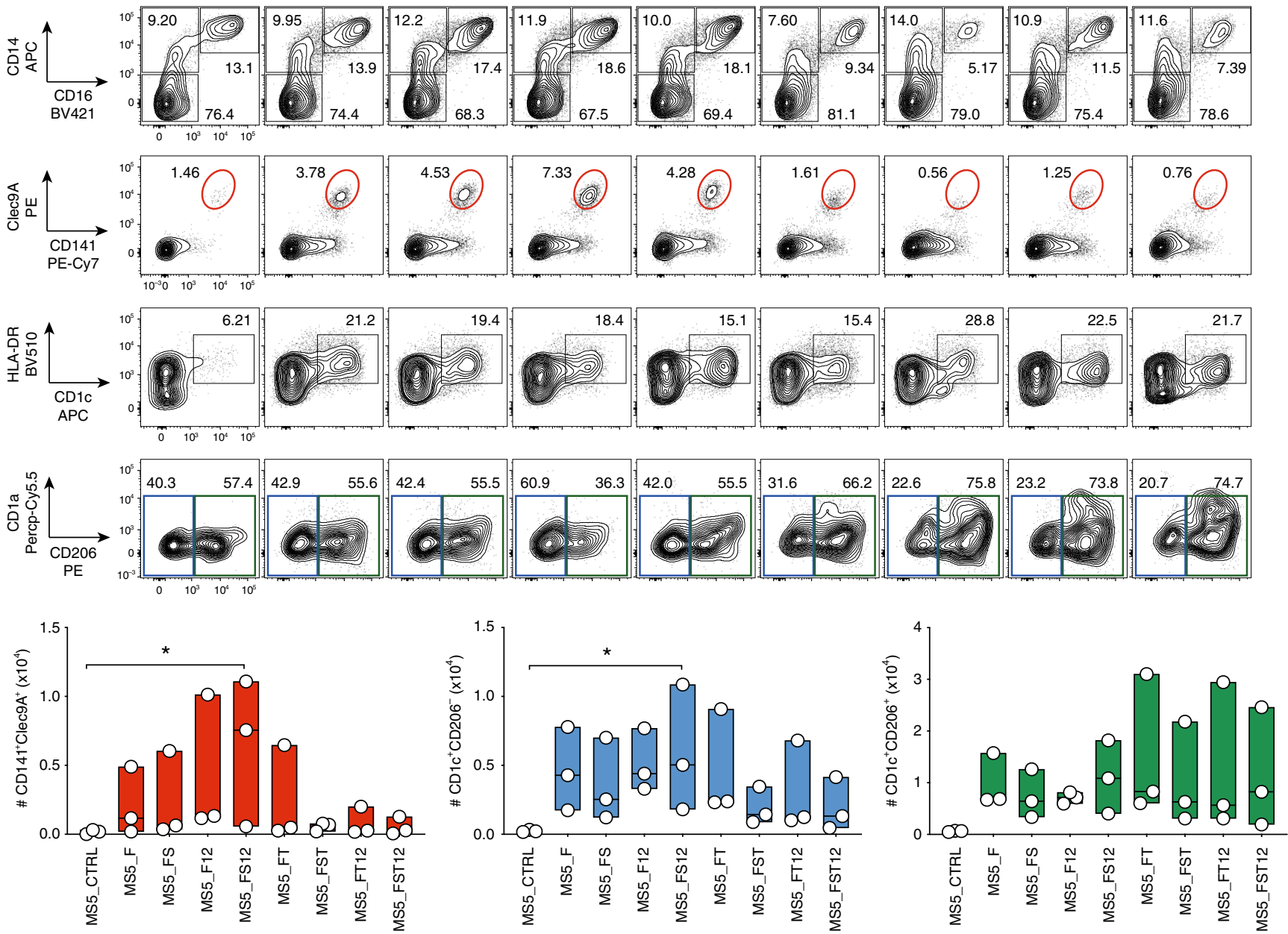

b

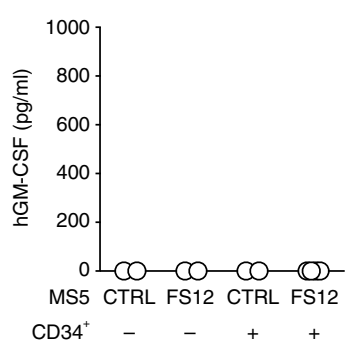

C

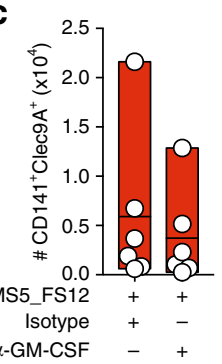

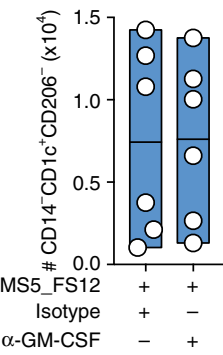

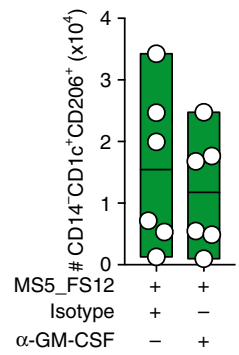

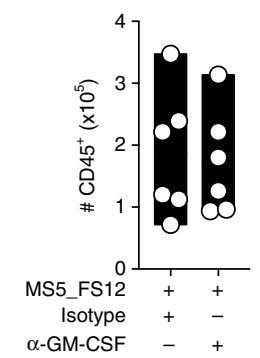

Fig. 2 CXCL12 and SCF improve FLT3L-driven DC differentiation. a Representative FACS plots and absolute number of CD141+Clec9A ${ }^{+}, \mathrm{CD}^{+} \mathrm{C}^{+} \mathrm{CD} 206^{-}$, and $\mathrm{CD} 1 \mathrm{c}^{+} \mathrm{CD}_{206}{ }^{+}$cells generated from CD34 ${ }^{+} \mathrm{HSPC}$ s cultured with MS5 expressing human FLT3L (MS5_F) in combination with human SCF (S), TPO (T), and CXCL12 (12). Day 15 flow cytometry analysis of $n=3$ cord blood donors in three independent experiments. ${ }^{*} p<0.05$, one-way ANOVA test with Tukey's multiple comparisons. b ELISA detecting human GM-CSF in the supernatant of CD34+ HSPCs cultured with engineered MS5 expressing human FLT3L, SCF and CXCL12 (MS5_FS12) at day 15 for $n=2$ (MS5_CTRL \pm CD34+ HSPC and MS5_FS12 only) and $n=4$ (MS5_FS12 + CD34+ HSPC) independent donors. c Absolute number of human DC subsets generated in vitro from CD34+ HSPC using MS5_FS12 stromal cells in the presence of human GM-CSF neutralizing antibody as compared to isotype control ( $n=6$ independent donors in two experiments). Data are presented as floating bars ranging from min to max and line represents median $\mathbf{a}, \mathbf{c}$ or as bar graphs with mean $\pm \mathrm{SEM}$ (b).

cultures containing eMSC co-expressing membrane-bound SCF and CXCL12 together with FLT3L (MS5_FS12) (Fig. 2a), whereas no difference was observed for $\mathrm{CD} 14^{+} \mathrm{CD} 16^{-}$monocytes and CD14 ${ }^{+} \mathrm{CD} 16^{+}$macrophages as compared to MS5_CTRL (Supplementary Fig. $3 b)$.
Furthermore, we noticed that in vitro differentiated CD14 CD1c ${ }^{+} \mathrm{cDC} 2$-like cells were heterogeneous for the expression of the mannose receptor CD206 (Fig. 2a). Circulating blood cDC2s do not generally express CD206 (Supplementary Fig. 3c), whereas $\mathrm{CD} 206$ is a marker of skin and migratory $\mathrm{CDC} 2^{19,55}$. 
Most of the previously described protocols to generate human DC-like cells in vitro from both $\mathrm{CD} 14^{+}$monocytes and $\mathrm{CD} 34^{+}$HSPCs made an extensive use of the cytokine GM$\mathrm{CSF}^{7,8,36,38,39,54}$, with one exception ${ }^{47}$. As we did not include GM-CSF in our protocol, we wanted to assess whether human GM-CSF was spontaneously produced in $\mathrm{CD} 34^{+}$cultures. We could not detect any GM-CSF from co-culture supernatant (Fig. 2b). Accordingly, GM-CSF-blocking antibody did not impact the generation of cDCs driven by MS5_FS12 (Fig. 2c). We conclude that GM-CSF is dispensable for the generation of cDCs in vitro, as previously reported in both mouse ${ }^{56,57}$ and human $^{47}$.

We also observed that MS5_FS12 stromal cells significantly improve the differentiation of $\mathrm{CD} 123^{+} \mathrm{CD} 303 / 4^{+}$cells (Fig. $3 a$ ), a phenotype shared by both plasmacytoid DC and pre/AS-DC ${ }^{12,20}$. A more refined phenotypic characterization of the in vitrogenerated cells also shows that all $\mathrm{CD} 123^{+}$cells express high levels of CD45RA and they can be subdivided in $\mathrm{AXL}^{-} \mathrm{CD} 327 \mathrm{lo}-$ and $\mathrm{AXL}^{+} \mathrm{CD}_{2} 7^{+}$subsets, phenotypically aligning to $\mathrm{pDC}$ and pre/AS-DC (Fig. 3b). This conclusion was further supported by gene set enrichment analysis (GSEA $)^{58}$ of RNA-sequencing (RNA-seq) data, displaying a significant enrichment of previously reported $\mathrm{pDC}$ and AS-DC gene signatures ${ }^{20}$ in in vitro-generated $\mathrm{AXL}^{-} \mathrm{CD} 327^{\mathrm{lo} /}-$ and $\mathrm{AXL}^{+} \mathrm{CD}_{2} 7^{+}$, respectively (Fig. $3 \mathrm{c}$ ). Moreover, only the $\mathrm{AXL}^{-} \mathrm{CD} 327^{\mathrm{lo}}-$ - cells were capable to produce type I interferon in response to toll-like receptor (TLR) stimulation, a specific feature of bona fide $\mathrm{pDC}$, which is not shared with pre/AS-DC ${ }^{12,20}$ (Fig. 3d).

In conclusion, we identified the combination of membranebound FLT3L, SCF, and CXCL12 (MS5_FS12) as the most efficient tested condition to support human DCs differentiation in vitro from $\mathrm{CD} 34^{+}$HSPCs.

Human DC generated in vitro align with circulating blood DC. To validate the identity of the $\mathrm{cDCs}$ generated using the MS5_FS12 stromal niche, we compared the transcriptome (RNAseq) and phenotype (CyTOF) of in vitro-differentiated subsets to circulating blood cDC1s and cDC2s (Fig. 4a-f).

Hierarchical clustering of RNA-seq data revealed that subsets generated in culture maintain a strong "culture imprinting" (Supplementary Fig. 4a). Indeed, we could identify a 2000 genes signature (1000 genes up- and 1000 genes downregulated), which clearly separates in vitro-generated cells from circulating blood subsets regardless of their subset identity (Supplementary Fig. 4b). The majority of these genes were associated to cell cycle and metabolism as shown by pathways enrichment analysis (Supplementary Fig. 4c). Nonetheless, once this "in vitro culture signature" was subtracted from the total protein coding genes, $\mathrm{CD}_{141}{ }^{+} \mathrm{Clec} 9 \mathrm{~A}^{+}$and $\mathrm{CD} 1 \mathrm{c}^{+} \mathrm{CD} 206^{+/-}$cells generated in culture transcriptionally align to blood $\mathrm{cDC1}$ and $\mathrm{cDC} 2$, respectively (Fig. 4a).

To further validate the similarity of in vitro generated cells with physiologically circulating subsets, we performed GSEA $^{58}$ using the BubbleGum software ${ }^{59}$. This methodology enables to score the enrichment of a signature in a pairwise comparison of two transcriptomes. We scored $\mathrm{cDC} 1$ alignment using gene signatures specific for blood $\mathrm{CDC1}$ obtained from published datasets $\left(\mathrm{cDC} 1>\mathrm{CD} 1 \mathrm{c}^{[+60}\right.$ and $\left.\mathrm{DC} 1>\mathrm{ALL}^{20}\right) . \mathrm{CD}^{-} 4^{-} \mathrm{CD} 1 \mathrm{c}^{+}$cells have recently been shown to contain two distinct subsets termed as $\mathrm{cDC} 2$ and $\mathrm{CDC}^{20}$. Alignment of cultured cells was probed towards total CD1c ${ }^{+}$cells $(\mathrm{CD} 1 \mathrm{c}>\mathrm{cDC} 1), \mathrm{cDC} 2(\mathrm{cDC} 2>\mathrm{ALL}$ and $\mathrm{cDC} 2>\mathrm{DC} 3)$, and $\mathrm{DC} 3$ signatures $(\mathrm{DC} 3>\mathrm{cDC} 2$ and $\mathrm{DC} 3>\mathrm{ALL})$. We found that in vitro-generated $\mathrm{CD} 141^{+} \mathrm{Clec} 9 \mathrm{~A}^{+}$and $\mathrm{CD} 1 \mathrm{c}^{+}$ CD206 $6^{+/}-$cells are enriched in genes defining circulating blood $\mathrm{cDC} 1$ and $\mathrm{cDC} 2$, respectively (Fig. $4 \mathrm{~b}$ ). The expression of the top
50 genes for each signature in the differentiated subsets further supports this conclusion (Fig. 4c). Importantly, both CD $206^{+}$and $\mathrm{CD}^{206^{-}}$subsets aligned preferentially to $\mathrm{CDC} 2$ as compared with DC3 and cDC1 (Fig. 4b and Supplementary Fig. 4d). CD163 was recently described as a marker selectively expressed in blood cDC3 as compared with $\mathrm{cDC} 22^{20}$. Supporting our previous conclusion, CD163 was neither expressed in $\mathrm{CD}^{+} \mathrm{c}^{+} \mathrm{CD} 206^{-}$ nor in $\mathrm{CD}_{1 \mathrm{c}^{+}} \mathrm{CD} 206^{+}$cells generated in vitro, whereas $\mathrm{CD} 163^{+}$ cells were detected among $\mathrm{CD} 14^{+}$monocytes and $\mathrm{CD} 14^{+} \mathrm{CD} 16^{+}$ macrophages (Supplementary Fig. 4e).

To obtain a more exhaustive characterization of the phenotype of in vitro-generated subsets, we performed CyTOF analysis using a panel of 38 metal-conjugated monoclonal antibodies. Dimension reduction of the CyTOF data was performed using the Uniform Manifold Approximation and Projection (UMAP) algorithm ${ }^{61}$. UMAP plots display clusters of cells that were expanded upon MS5_FS12 co-culture as compare to MS5_CTRL (Fig. 4d). Clec9A ${ }^{+} \mathrm{CD}_{141}{ }^{+}$cells identified by flow cytometry were shown to also express CADM1 and CD26 further aligning them with blood cDC1s (red cluster, Fig. $4 d-f$ ). CD14-CD1c ${ }^{+}$ cells did not express high level of monocyte markers such as CD64, CD68, and CD16, whereas they appeared heterogeneous for CD206 expression (blue cluster, Fig. 4d-f and Supplementary Fig. 4f). Of note, $\mathrm{CD} 14^{-} \mathrm{CD} 1 \mathrm{c}^{+}$cells generated in culture did not express high level of FceRIa, CD172a, and CD5 found in blood cDC2s (Supplementary Fig. 4f). By contrast, they were strongly positive for CD86 and CD80 unlike their circulating counterpart (Supplementary Fig. 4f). In addition, CD123 ${ }^{+} \mathrm{CD} 303^{+}$cells were shown to express heterogeneous levels of pre-DC markers such as CD327 and CX3CR1, and moderate level of AXL (green cluster, Fig. 4d-f and Supplementary Fig. 4f), in line with flow cytometry analysis highlighting the presence of both $\mathrm{pDC}$ and pre/AS-DC within $\mathrm{CD} 123^{+} \mathrm{CD} 33^{+}$cells generated in vitro (Fig. $3 \mathrm{~b}$ ). On the other hand, by combining flow and mass cytometry analysis, we were able to show that MS5_FS12 stromal cells do not support lymphoid development (Fig. 4d and Supplementary Fig. 4g). Indeed, the remaining cells (other than DC) present in culture consist of $\mathrm{CD} 15^{+}$Granulocytes and CD14 ${ }^{+} \mathrm{CD} 16^{+/-}$Monocytes/ Macrophages (Supplementary Fig. 4h). Finally, the analysis of in vitro $\mathrm{cDC}$ differentiation kinetics revealed that both $\mathrm{CDC} 1$ and cDC2 can be detected in MS5_FS12 cultures as early as day7 (Supplementary Fig. 4i). However, the yield of in vitro generated cDC was significantly higher at day14, when most of the cultures were therefore analyzed (Supplementary Fig. 4i).

Collectively, our data demonstrate that: (i) in vitro-generated $\mathrm{CD} 141^{+} \mathrm{Clec} 9 \mathrm{~A}^{+}$recapitulate the phenotype of bona fide blood cDC1; (ii) $\mathrm{CD}_{14}{ }^{-} \mathrm{CD} 1 \mathrm{c}^{+}$cells align to $\mathrm{cDC} 2$ regardless of their CD206 expression; (iii) $\mathrm{CD} 123^{+} \mathrm{CD} 303^{+}$cells contain some recently described pre-DC/AS-DC phenotypically and functionally distinct from pDCs. However, we identified two major limitations of the in vitro culture. First, the culture system imposes a strong transcriptional imprinting throughout subsets. Second, in vitro-generated $\mathrm{cDC} 2 \mathrm{~s}$ failed to express to full phenotypic profile of blood $\mathrm{cDC} 2 \mathrm{~s}$.

Engineered stromal niches support HSPC maintenance in vivo. We next wanted to assess whether we could use MS5_FS12 to recapitulate a more physiological niche microenvironment supporting human HSPCs maintenance in vivo.

To this end, we designed an experimental strategy based on the subcutaneous injection of cord blood-derived CD34 HSPCs together with MS5_FS12 in a basement membrane matrix (Matrigel) in NOD.Cg-Prdc scid $\mathrm{Il} 2 \mathrm{rg}^{\mathrm{tm} 1 \mathrm{Wjl}} / \mathrm{SzJ}$ (NSG) mice (Fig. 5a). 
a
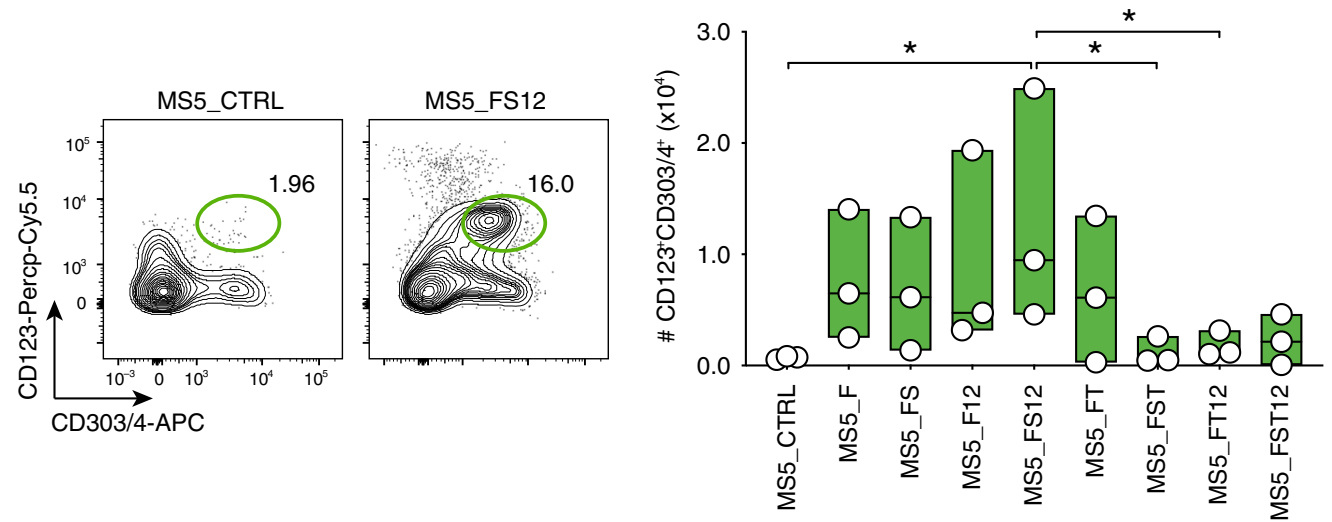

b
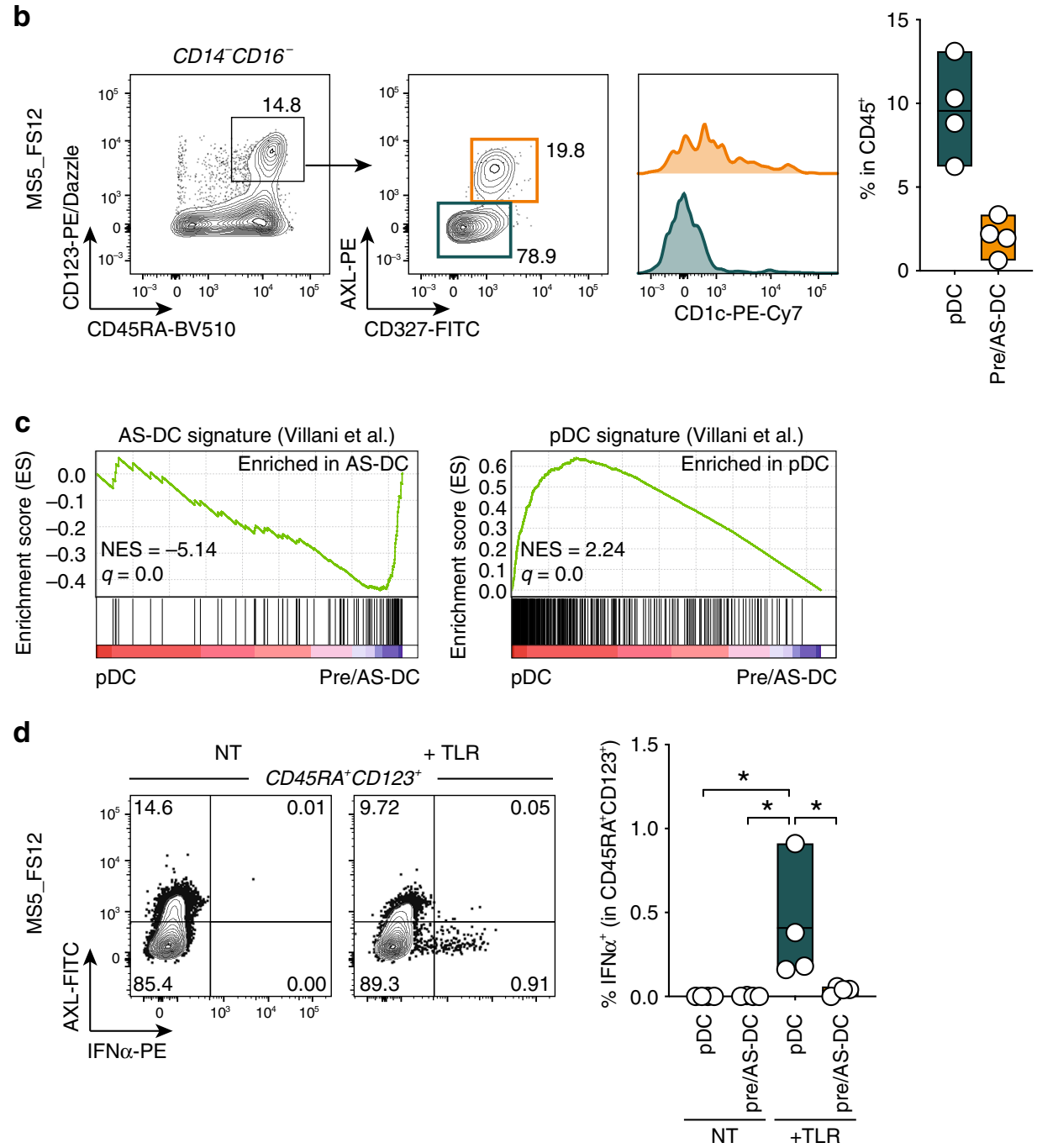

Fig. 3 MS5_FS12 stromal cells support pDC and pre/AS-DC development in vitro. a Representative FACS plots and absolute number of CD123+CD303/ $4^{+}$cells generated in vitro from CD34+ HSPCs co-cultured with MS5 expressing human FLT3L (MS5_F) in combination with human SCF (S), TPO (T), and CXCL12 (12). Day 15 flow cytometry analysis of $n=3$ cord blood donors in three independent experiments. * $p<0.05$, one-way ANOVA test with Tukey's

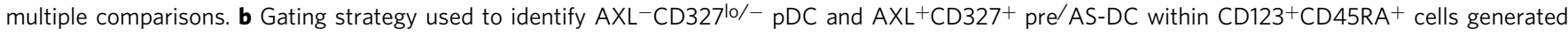
in vitro using MS5_FS12. Graph illustrates the frequency of each subset in CD45+ cells ( $n=4$ cord blood donors). c GSEA comparing in vitro-differentiated pDC and pre/AS-DC using published human $\mathrm{PDC}$ and AS-DC gene signatures ${ }^{20}$ (FDR false detection rate, NES normalized enrichment score). Statistical significance is defined by the FDR $q$-value calculated by the GSEA software (www.broad.mit.edu/gsea) using default parameters. $\mathbf{d}$ Intracellular flow cytometry analysis of IFN $\alpha$ production in pDC and pre/AS-DC in response to $16 \mathrm{~h}$ of TLR stimulation (lipopolysaccharide (LPS) $10 \mathrm{ng} / \mathrm{ml}, \mathrm{R} 8481 \mu \mathrm{g} / \mathrm{ml}$, Poly (I:C) $25 \mu \mathrm{g} / \mathrm{ml}$ ). Bar graph shows the frequency of IFN ${ }^{+}$cells in each subset with ( + TLR) or without (NT) stimulation ( $n=4$ cord blood donors). ${ }^{*} p<$ 0.05 , one-way ANOVA test with Holm-Sidak's multiple comparisons. Data are presented as floating bars ranging from min to max and line represents median (a, b, d). 
a

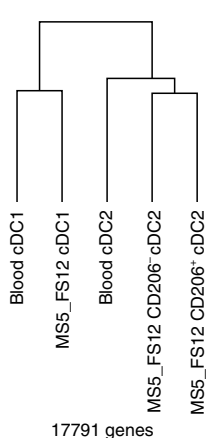

b

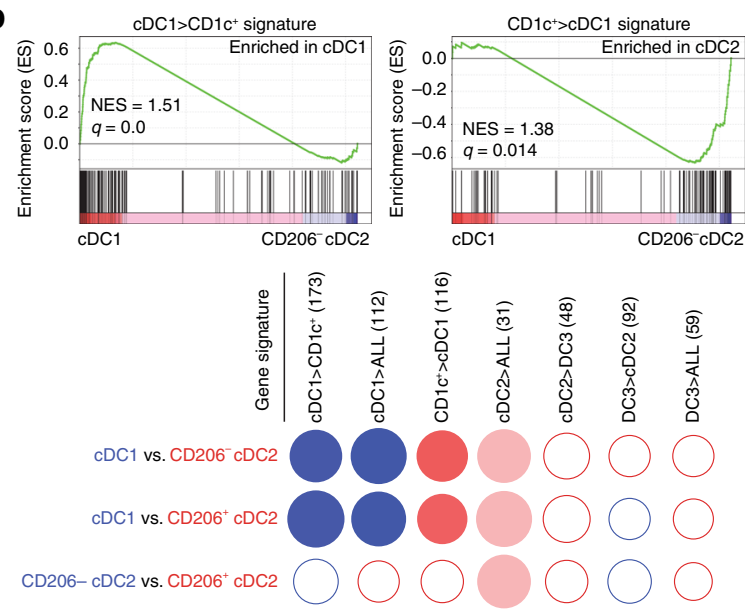

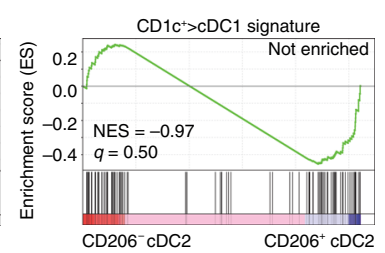

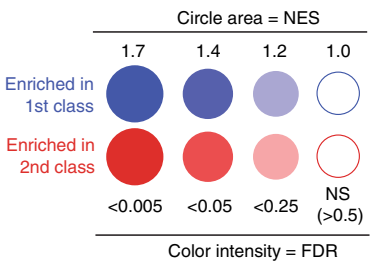

C

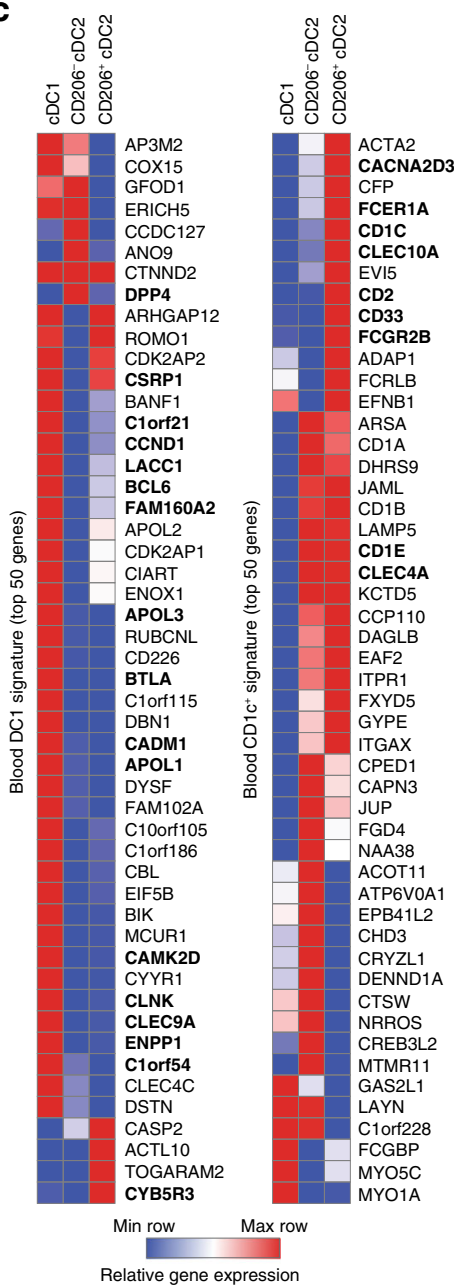

d

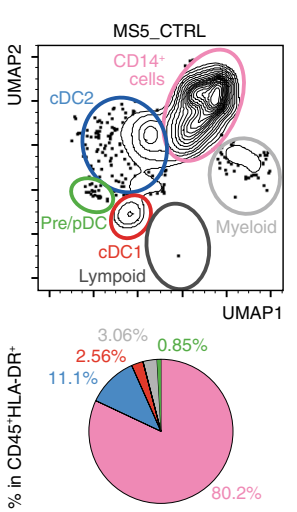

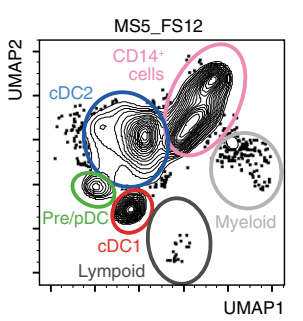
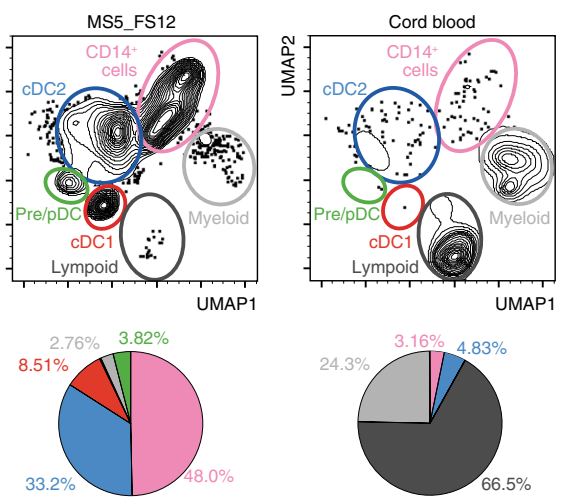

e
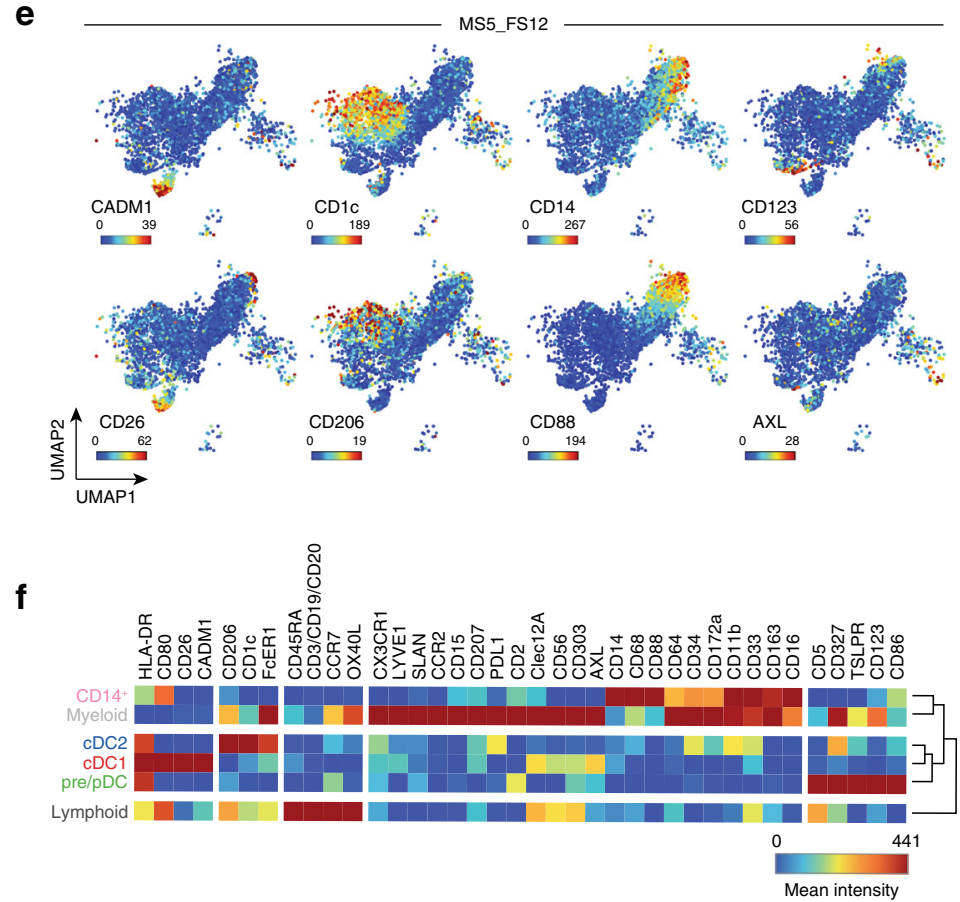

Clusters of cells embedded in Matrigel can be identified as early as day 12 by tissue histology (Fig. 5b). Flow cytometry analysis demonstrated that MS5_FS12 but not MS5_CTRL induced the expansion of human leukocytes within the Matrigel plugs (Fig. 5c). We then tested whether cell-to-cell interactions of eMSC with human progenitors play a role in this process. We injected two independent plugs of $\mathrm{CD} 34^{+}$HSPCs with either MS5_CTRL or MS5_FS12 in the same recipient mouse (contralateral plugs) (Fig. 5d). We found a relative expansion of human leukocytes in MS5_FS12 as compared to MS5_CTRL contralateral plugs (Fig. 5d). We conclude that MS5_FS12 does not efficiently provide soluble factors enabling human leukocytes 
Fig. 4 Human DC generated in vitro align with circulating blood DC. a Hierarchical clustering of primary ( $n=3$ healthy donors) vs. in vitro-generated ( $n=3$ cord blood donors) cDCs based on 17,791 genes after removing the "in vitro culture signature" (2000 genes) defined by pairwise comparison of primary versus in vitro generated subsets. b GSEA using blood $\mathrm{cDC1s}\left(\mathrm{DC} 1>\mathrm{CD} 1 \mathrm{c}^{+}\right)$and $\mathrm{CD1} \mathrm{c}^{+}$cells $\left(\mathrm{CD} 1 \mathrm{c}^{+}>\mathrm{DC} 1\right)$ signatures generated from published datasets 60 , as well as previously published signatures of blood cDC1 (DC1>ALL), cDC2 (DC2>ALL), and cDC3 (DC3>ALL) ${ }^{20}$. BubbleMap shows the enrichment of each gene signature in the pairwise comparison of $\mathrm{CD} 141^{+} \mathrm{Clec} 9 \mathrm{~A}^{+}, \mathrm{CD} 1 \mathrm{c}^{+} \mathrm{CD} 206^{-}$, and $\mathrm{CD} 1 \mathrm{c}^{+} \mathrm{CD} 206^{+}$cells generated in vitro $(\mathrm{FDR}$ false detection rate, NES normalized enrichment score). For single pairwise comparisons (top), statistical significance is defined by the FDR $q$-value calculated by the GSEA software (www.broad.mit.edu/gsea) using default parameters. For multiple pairwise comparisons (bottom), the statistical significance was further corrected for multiple testing by the BubbleMap module of BubbleGUM software. $\mathbf{c}$ Heatmaps of RNA-seq data displaying the expression of the top

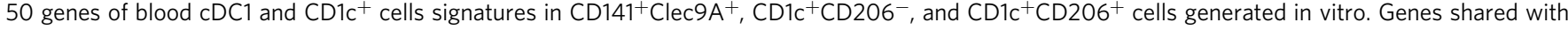
previously published signatures ${ }^{20}$ are highlighted in bold. d UMAP (Uniform Manifold Approximation and Projection) plots of CyTOF data from CD45 ${ }^{+} \mathrm{HLA}_{-} \mathrm{DR}^{+}$cells differentiated in vitro using MS5_FS12 and MS5-CTRL as compared with cord blood PBMCs. Pie charts indicate the frequency of each subset among the CD45+HLA-DR ${ }^{+}$cells (mean of $n=2$ cord blood donors in two independent experiments). e Relative expression of selected markers in UMAP plots of CyTOF data from cells differentiated in vitro with MS5_FS12. $\mathbf{f}$ Heatmap of markers mean intensity in each subset identified in MS5_FS12 cultures.

expansion systemically. Therefore, we hypothesized that membrane-bound FLT3L and SCF together with CXCL12 define an efficient in vivo niche by delivering cell-to-cell contacts supporting HSPCs expansion. Supporting this hypothesis, we found that MS5_FS12 expressing GFP persist in the plugs at day 12 of differentiation (Fig. 5e). Immunofluorescence analysis further supported this observation and demonstrated the existence of cell-to-cell contact between MS5_FS12 and human CD45 ${ }^{+}$leukocytes (Fig. 5e and Supplementary Fig. 5a). Leukocytes expressing $\mathrm{CD} 34^{+}$could also be detected, supporting the notion that a pool of undifferentiated progenitors is maintained in the MS5_FS12 organoids at day 12 (Fig. 5e). Of note, Matrigel plugs contained mouse $\mathrm{CD} 31^{+}$cells, suggesting undergoing vascularization as evidenced by the formation of early tube-like structure (Fig. 5f). However, no vascular leak was observed, as demonstrated by the absence of intravenously delivered $\mathrm{CTV}^{+} \mathrm{CD}^{+}$cells in the subcutaneous plug (Supplementary Fig. 5b).

Taken together, these data show that engineered stromal cells MS5_FS12 provide a minimal synthetic niche scaffold supporting human $\mathrm{CD}_{3}{ }^{+}$HSPCs maintenance and expansion in vivo.

The MS5_FS12 niche supports human DC development in vivo. We investigated whether the engraftment of $\mathrm{CD} 34^{+}$ HSPCs together with MS5_FS12 could lead to the local differentiation of human DC subsets.

Flow cytometry analysis of Matrigel organoids demonstrates that the MS5_FS12 but not the MS5_CTRL niche specifically supports the differentiation of $\mathrm{CD} 141^{+} \mathrm{Clec} 9 \mathrm{~A}^{+} \mathrm{cDC1}$-like cells and $\mathrm{CD} 14^{-} \mathrm{CD} 1 \mathrm{c}^{+} \mathrm{cDC} 2$-like cells (Fig. 6a and Supplementary Fig. 6a). This finding was supported by immunofluorescence staining on plug sections highlighting the occurrence of human CD45 cells expressing either Clec9A or CD1c (Fig. 6b).

Further analysis revealed the expansion of $\mathrm{CD} 123^{+} \mathrm{CD} 303 / 4^{+}$ cells in MS5_FS12 when compared to MS5_CTRL plugs (Fig. $6 \mathrm{c}$ and Supplementary Fig. 6a). All these cells also expressed CD45RA and heterogeneous levels of AXL and CD327, as previously described for their in vitro counterparts (Fig. 6c). However, only MS5_FS12 induced a strong accumulation of $\mathrm{AXL}^{+} \mathrm{CD} 327^{+}$pre/AS-DC expressing various levels of CD1c (Fig. 6c). In addition, bona fide $\mathrm{CD} 123^{+} \mathrm{CD} 45 \mathrm{RA}^{+} \mathrm{AXL}{ }^{-} \mathrm{CD} 327^{\mathrm{lo} /-}$ pDCs could also be detected (Fig. 6c). RNA-seq analysis of in vivo-generated $\mathrm{CD} 123^{+}$ $\mathrm{AXL}^{-} \mathrm{CD} 327^{\mathrm{lo} /}-$ and $\mathrm{CD}^{-} 23^{+} \mathrm{AXL}^{+} \mathrm{CD} 327^{+}$cells further support this conclusion and unequivocally align them to blood circulating pDC and AS-DC, respectively (Fig. 6d).

To further refine the phenotypic characterization of HLA-DR ${ }^{+}$ mononuclear phagocytes in MS5_FS12 organoids we performed high-dimensional mass cytometry analysis. The comparison of MS5_FS12 with MS5_CTRL plugs highlighted the expansion of all subsets previously identified by flow cytometry: $\mathrm{cDC} 1 \mathrm{~s}, \mathrm{cDC} 2 \mathrm{~s}$, pre/AS-DCs, pDCs, and a distinct population of $\mathrm{CD}_{3}{ }^{+}$ CCR2 ${ }^{+} \mathrm{CX} 3 \mathrm{CR} 1{ }^{+} \mathrm{Clec} 12 \mathrm{~A}^{+}$myeloid cells (Fig. 6e and Supplementary Fig. 6b). We next wanted to determine whether commitment towards the $\mathrm{cDC}$ lineage would be dependent on local developmental cues and possibly cell-to-cell contact between CD34 ${ }^{+}$HSPCs and MS5_FS12. To this end, we engrafted mice with two distal organoids, one containing MS5_CTRL and the second one containing MS5_FS12. We found that cDC1, cDC2, and pre/AS-DCs were selectively expanded in MS5_FS12 plugs (Fig. $6 f$ and Supplementary Fig. 6c). On the contrary, pDC were not significantly increased in the same comparison (Fig. $6 \mathrm{f}$ and Supplementary Fig. 6c). We conclude that local cues associated to the MS5_FS12 niche control cDC lineage commitment. In support of this view, we could not detect a systemic increase in the levels of serum FLT3L in mice carrying engineered stromal cell plugs (Fig. 6g). Accordingly, spleen resident murine cDCs did not expand upon MS5_FS12 engraftment, while they massively do so upon administration of recombinant soluble human FLT3L (Fig. 6g). Together with the two-plugs experiments (Fig. 6f), these observations suggest that most of the FLT3L aegis relies on its membrane-bound form delivered in the context of eMSCs. Of note, administration of recombinant soluble FLT3L was poorly efficient at expanding human DCs populations in Matrigel organoids formed with control stromal cells (Fig. 6g). This demonstrates the superiority of local, cell-associated cues (i.e., MS5_FS12) to achieve the expansion of human cDCs in the dermis of NSG mice.

A more extensive characterization of MS5_FS12 organoids revealed the presence of myeloid lineages other than DCs, such as $\mathrm{CD} 14^{+} \mathrm{CD} 16^{-}$monocyte-like cells and $\mathrm{CD} 15^{+}$granulocytes (Fig. 6h and Supplementary Fig. 6d). Conversely, no lymphoid specification was observed (Fig. 6h and Supplementary Fig. 6d). Despite this broad spectrum of lineages, CD34 ${ }^{+}$HSPCs represented the most abundant population at day 12 (Fig. 6h). This observation suggests that the MS5_FS12 niche combines HSPC maintenance with lineage commitment.

cDC2 generated in vivo faithfully align to blood cDC2. Finally, we wanted to establish whether in vivo differentiated DCs in MS5_FS12 organoids had a distinct phenotype from the subsets generated in vitro in MS5_FS12 co-culture.

UMAP plots of CyTOF analysis revealed three major findings. First, pre/AS-DCs represent a more abundant population in vivo (Fig. 7a and Supplementary Fig. 7a). Second, both cDC1 generated in vitro and in vivo fully align phenotypically, displaying a strong expression of CADM1 and CD26 (Fig. 7a and Supplementary Fig. 7a). Third, unlike cDC1, cDC2 generated in vivo exhibit noticeable phenotypic differences. In vivo-generated cDC2 express 
a

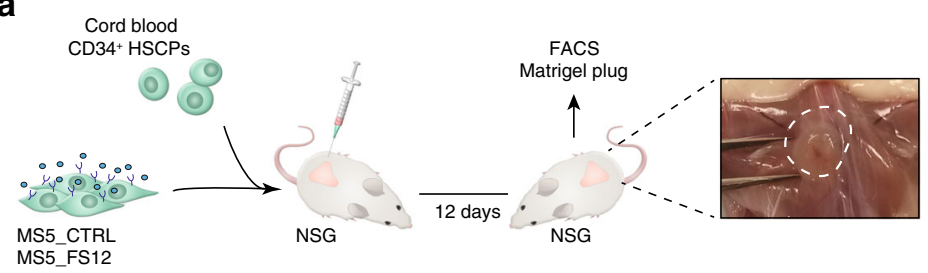

b

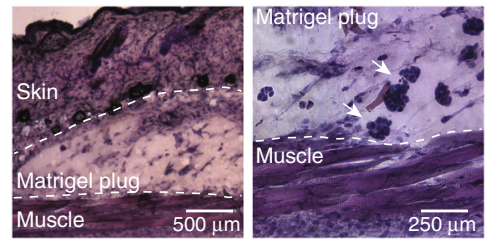

C
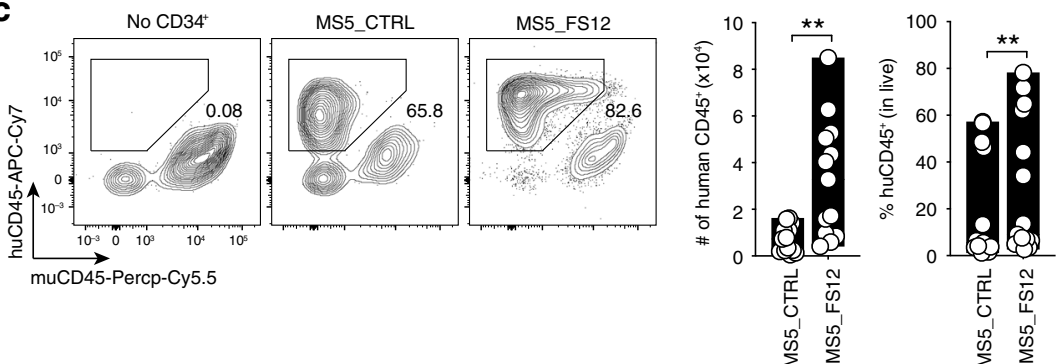

d
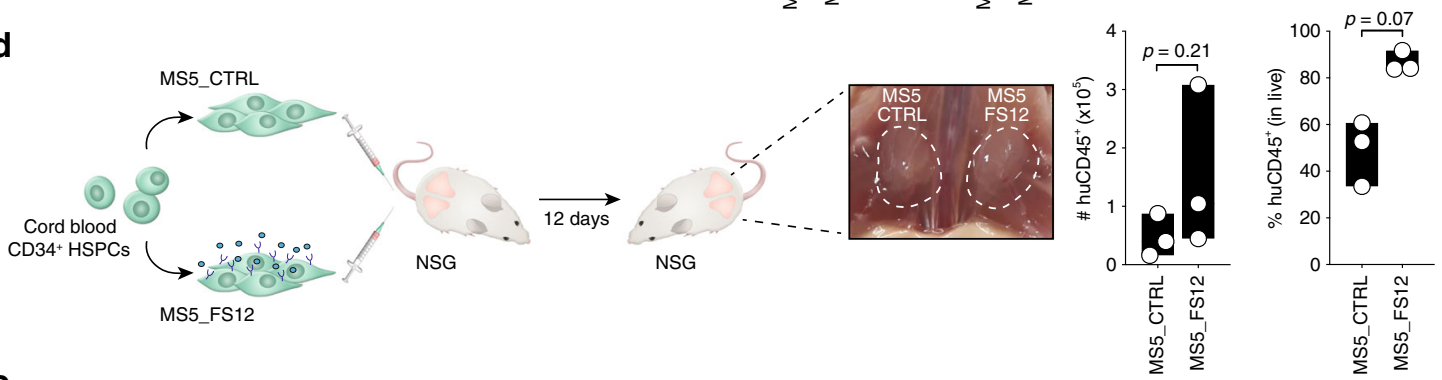

e
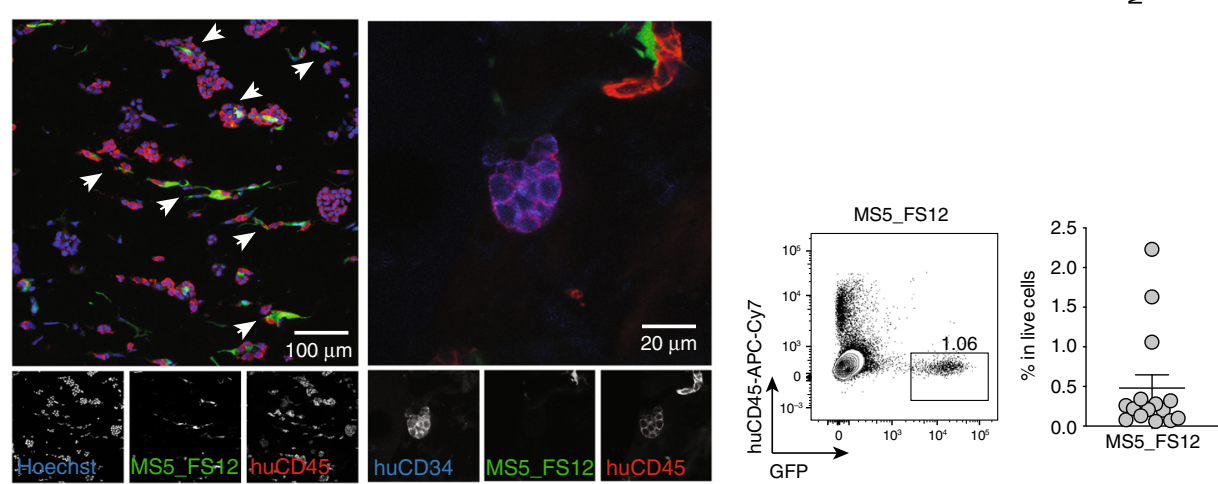

f
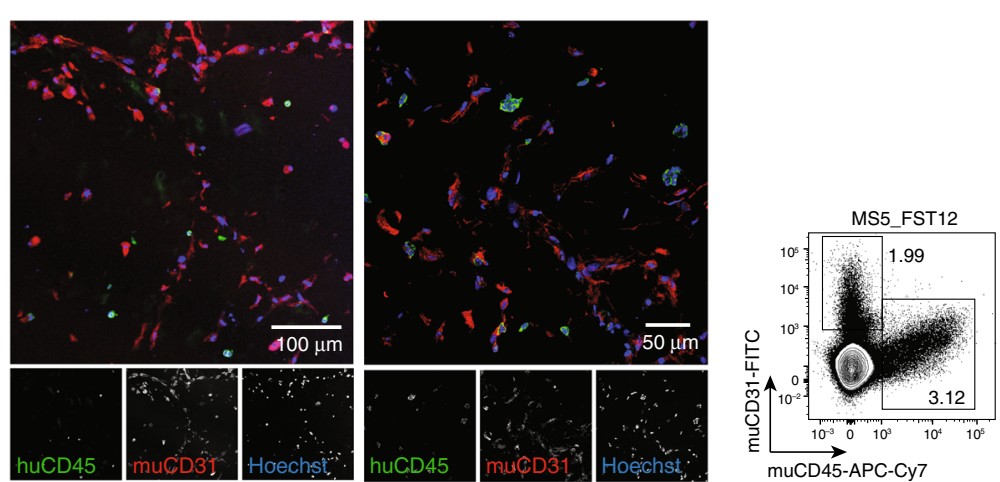

higher levels of FceRIa, CD172a, and CD5, while showing lower expression of HLA-DR and CD86 (Fig. 7b and Supplementary Fig. 7b). The specific phenotype conferred by the MS5_FS12 niche education renders $\mathrm{CDC} 2 \mathrm{~s}$ more akin to their blood counterparts. In order to compare extensively the transcriptional landscape of in vivo (NSG organoids) generated DCs with primary DCs found in human blood, we performed RNA-seq analysis on fluorescence- activated cell sorting (FACS)-sorted cDC2s obtained after the enzymatic digestion of MS5_FS12-containing plugs or purified from human blood.

As previously observed for in vitro-generated cells, the MS5_FS12 niche confers an in vivo imprinting resulting in the differential expression of 2872 genes (up- or downregulated) in in vivo versus ex vivo-isolated subsets (Supplementary Fig. 7c). 
Fig. 5 Engineered stromal niches support HSPC maintenance in vivo. a Experimental strategy for an in vivo synthetic niche. Human HSPCs were injected subcutaneously along with MS5_FS12 in a basement membrane matrix (Matrigel) preparation. b Hematoxylin-eosin staining of subcutaneous organoids at day 12. Arrows show clusters of Matrigel-embedded cells. Scale bar represents $500 \mu \mathrm{m}$ (left) and $250 \mu \mathrm{m}$ (right). c Flow cytometry analysis at day 12 of Matrigel organoids containing either MS5_CTRL or MS5_FS12 cells. Absolute number and frequency of human CD45+ cells recovered are summarized in bar graphs $\left(n=13\right.$ cord blood donors in 6 independent experiments; ${ }^{\star \star} p<0.01$, two-tailed paired Student's $t$-test). d Experimental strategy and quantification of human CD45+ cells recovered from physically separated plugs containing either MS5_CTRL or MS5_FS12 cells injected in the same recipient ( $n=3$ cord blood donors in one experiment; two-tailed paired Student's $t$-test). e Immunofluorescence staining of plug sections displaying the interaction of GFP+ MS5_FS12 (green) with human CD45+ cells (red). Human hematopoietic progenitors were also identified as CD45 ${ }^{+}$(red) CD34 $^{+}$ (blue) cells in MS5_FS12 plugs. Nuclei were stained with Hoechst (blue). Arrows show interaction of human CD45+ leukocytes with GFP+ MS5_FS12. Scale bar represents $100 \mu \mathrm{m}$ (left panel) and $20 \mu \mathrm{m}$ (right panel). Similar results were observed in $n=5$ Matrigel organoids. The presence of GFP + stromal cells in Matrigel organoids at day 12 was further confirmed by flow cytometry ( $n=15$ independent organoids). $\mathbf{f}$ Visualization of mouse CD31 ${ }^{+}$endothelial cells by immunofluorescence. Fixed sections were stained for human CD45 (green) and mouse CD31 (red). Nuclei were stained with Hoechst (blue). Scale bar represents $100 \mu \mathrm{m}$ (left panel) and $50 \mu \mathrm{m}$ (right panel). Similar results were observed in $n=5$ Matrigel organoids. The presence of mouse CD31+ cells was further confirmed by flow cytomery. Data are presented as floating bars ranging from min to max and line represents median (c, d) or scatter plots with mean $\pm \operatorname{SEM}(\mathbf{e})$.

Pathway analysis revealed that this in vivo bias was mainly due to upregulation of genes associated with DNA replication, cell cycle and proliferation (MYC, CDC6/7, POLA2, MCM6/7) (Supplementary Fig. 7c and Supplementary Table 8).

Moreover, we found that: (i) $\mathrm{AXL}^{+}$Siglec6 ${ }^{+}$pre/AS-DCs generated in vivo (or in vitro) align to their primary counterparts and selectively express a signature that distinguish them from bona fide pDCs (DAB2, CD22, ADLH2) (Fig. 7c). (ii) conversely, $\mathrm{AXL}^{-}$Siglec6 ${ }^{-}$bona fide $\mathrm{pDCs}$ generated in vivo (or in vitro) align to their primary counterparts and express high levels of markers distinguishing them from pre/AS-DCs (IRF7, GZMB, TCF4, BCL11A) (Fig. 7c). (iii) cDC2s generated in vivo (in NSG mice organoids carrying MS5_FS12) had higher levels of similarity with blood cDC2s (including higher expression of BTLA, FCER1A) (Figs. 7b, d, e). Recently, both CD5 ${ }^{+}$and $\mathrm{CD}^{-}$ CDC2s subsets have been reported in human blood ${ }^{62,63}$ and we found that in vivo generated $\mathrm{CDC} 2 \mathrm{~s}$ aligned particularly well with blood $\mathrm{CD} 5{ }^{+} \mathrm{cDC} 2 \mathrm{~s}$ (with the expression of CD5, CD2) (Figs. 7b, e). By contrast, in vitro-generated cDC2s expressed high levels of activation genes such MHC molecules ( $H L A-D R, D Q)$, costimulatory molecules (CD80, CD40), activation markers (ETS2, CCR6, CCR7, CXCL13, CCL22) (Figs. 7e, f) and genes associated with type I and type II interferon pathways (STAT1, IRF9, IGS15, GBP1) (Supplementary Fig. 7d and Supplementary Table 9).

All together, we conclude that MS5_FS12-containing organoids provide a unique scaffold for the specification and commitment of the DC lineage. This unique and versatile system bypasses the limitation of in vitro cultures, which generated inefficiently pre/ AS-DCs and biased the differentiation of cDC2s toward an activated phenotype. Collectively, MS5_FS12 organoids faithfully recapitulate the differentiation of not only $\mathrm{pDCs}, \mathrm{cDC1}$, and cDC2s but also pre/AS-DCs, and support the development of cDC2s displaying an unattained level of similarity with their human blood counterparts.

In vitro and in vivo $\mathrm{cDC} 2$ functionally align to blood $\mathrm{cDC} 2$. In the last set of experiments, we aimed at functionally validate cord blood-derived cDC generated in the MS5_FS12 stromal niche. Moreover, we also assessed whether the phenotypic differences observed in $\mathrm{CDC} 2$ generated in vitro and in vivo may impact their function.

We first confirmed in vitro the responsiveness of cord bloodderived CDC2 to TLR agonists expressed in human circulating CDC2, as demonstrated by the upregulation of maturation markers (i.e., HLA-DR, CD86, and CD83) in response to TLR4 (LPS) and TLR8 (VTX-2337) stimulation (Fig. 8a). We then performed a mixed lymphocyte reaction (MLR) by co-culturing CTV-labeled allogeneic naive T cells together with FACS-sorted
cDC subsets (Supplementary Fig. 8a) activated overnight with a TLR agonists cocktail comprising of LPS (TLR4), R848 (TLR7/8) and Poly(I:C) (TLR3). After 7 days of culture, we observed that both in vitro and in vivo-generated $\mathrm{CDC} 2$ and pre/AS-DC were capable to efficiently induce $\mathrm{CD}^{+}$naive $\mathrm{T}$-cell proliferation (Fig. 8b), as expected and reported for circulating blood cDC2 $2^{12,20}$ (Supplementary Fig. 8b). Conversely, pDC were significantly less effective on triggering T-cell activation, as shown by the consistent reduction in the frequency of dividing $\mathrm{CD}^{+} \mathrm{T}$ cells when compared with $\mathrm{cDC} 2$ and pre/AS-DC (Fig. 8b).

Importantly, only cDC2 were able to produce high amounts of T-cell-polarizing cytokines in response to TLR stimulation, as demonstrated by intracellular TNFa and IL-12 detection by flow cytometry (Fig. 8c). All these features demonstrate that $\mathrm{cDC} 2 \mathrm{~s}$, pDCs and pre/AS-DCs functionally align to their in vivo counterparts as previously described in the literature ${ }^{12,20}$.

Collectively, our data suggest that: (i) both in vitro and in vivo differentiated $\mathrm{CDC} 2$ are equally capable to induce $\mathrm{CD} 4{ }^{+} \mathrm{T}$ cells activation and produce large amounts of TNF- $\alpha$ and IL-12; (ii) pre/AS-DC are as efficient as CDC2 in activating allogeneic naive $\mathrm{CD} 4+\mathrm{T}$ cells in vitro, a distinctive feature that clearly separate them from the pDC lineage; (iii) despite their ability to induce $\mathrm{CD}^{+}{ }^{+}$T-cell proliferation in MLR settings, pre/AS-DC do not produce high levels of cytokines commonly associated with $\mathrm{CDC} 2$ function, such as TNF- $\alpha$ or IL-12.

\section{Discussion}

Over the last two decades, DC-based strategies have been proposed for the therapeutic vaccination against cancer, including (i) non-targeted protein-based vaccines captured by DCs in vivo, (ii) specific targeting of DC subsets with mAb coupled to tumor antigens ${ }^{64}$ and (iii) antigen loading of ex-vivo-generated DCs ${ }^{3}$. In this context, experimental models recapitulating the development of human DC subsets are crucially needed.

Here we describe a novel approach to model human DC development from CD34 ${ }^{+}$HSPCs both in vitro and in vivo. To this end, we primarily focused on the physiological niches where human DCs differentiate and maintain: a central bone marrow niche where DC progenitors are specified and peripheral niches in the lymph nodes where DCs reside.

Previous studies have shown that the cell-to-cell interaction with membrane-bound factors expressed by the niche microenvironment plays an essential role in HSPCs maintenance and expansion ${ }^{21,22,65,66}$. Alternative splicing of human and murine SCF transcript results in the synthesis of both a soluble and a membrane-bound non-cleavable form of the protein. Interestingly, the secreted form of SCF/KITLG is not sufficient for the 
a

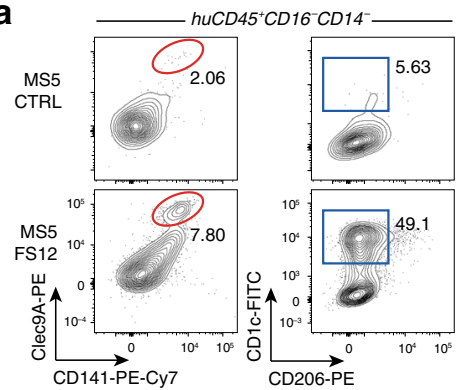

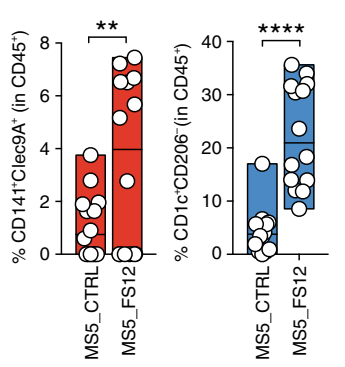

b

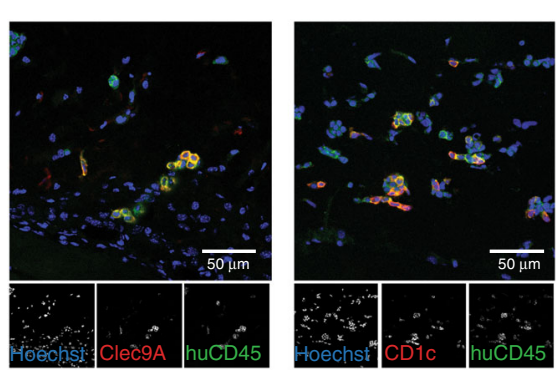

C
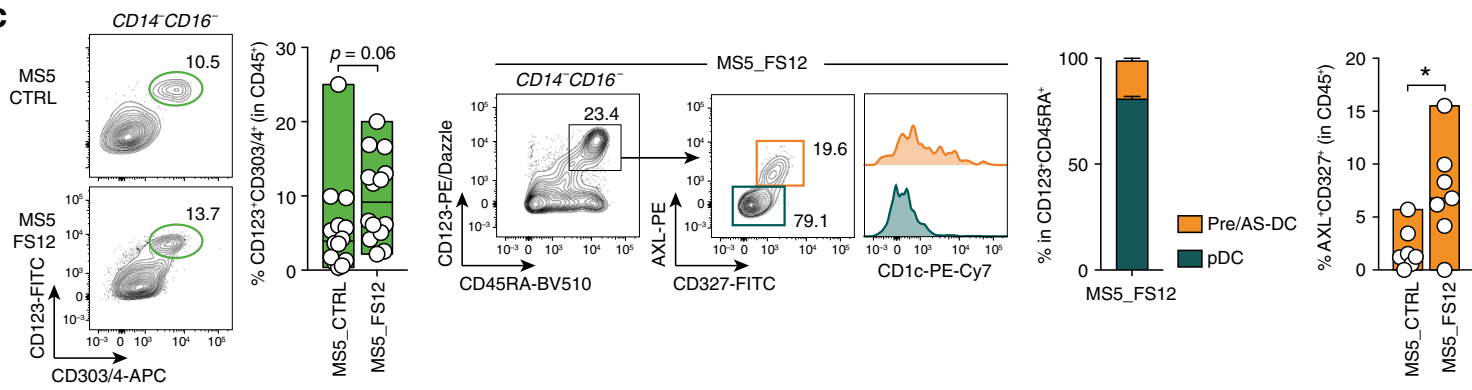

d
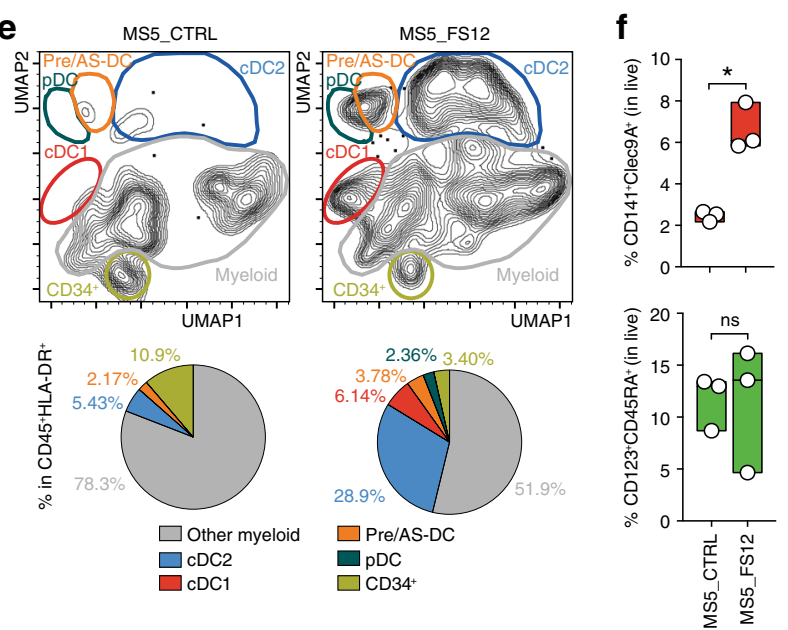

$\square \mathrm{CD} 34^{+}$

g

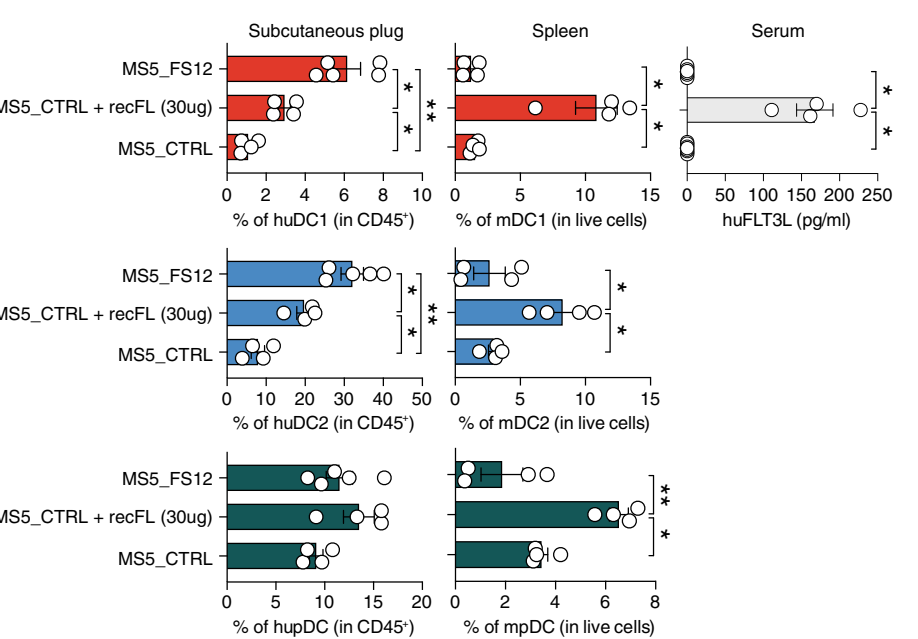

h
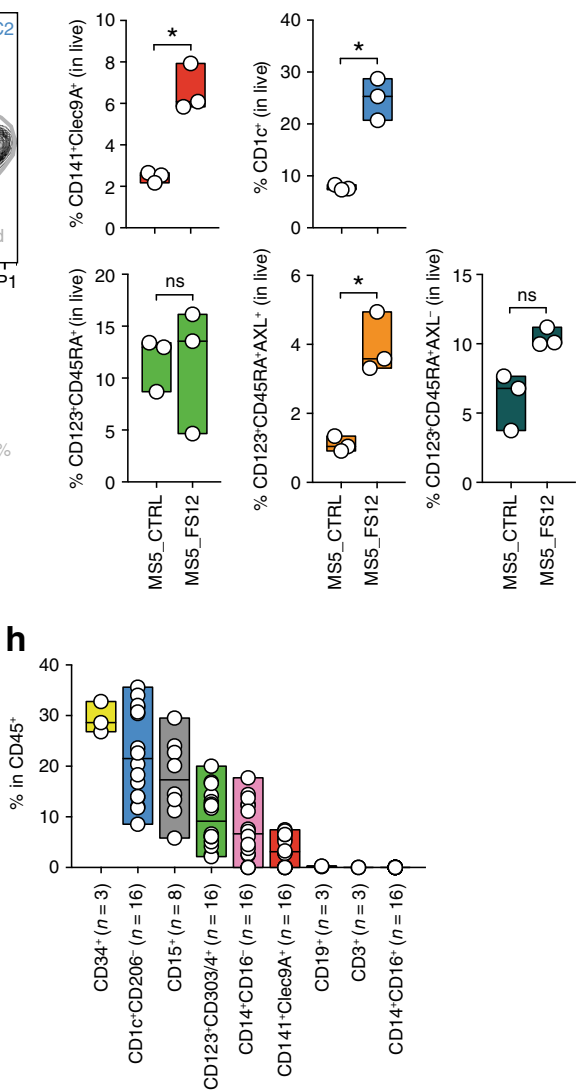

establishment of a functional niche in murine bone marrow ${ }^{21,22}$, whereas the expression of human membrane-bound SCF is sufficient to support human myeloid development in humanized mice ${ }^{66}$. We therefore wanted to test whether a similar relationship might exist between the soluble and membrane-bound forms of human FLT3L. Consistent with this hypothesis, the expression

of transmembrane FLT3L in mesenchymal stromal cells (MS5_F) improved the efficiency of DC differentiation in vitro, as compared to its soluble form (MS5 + recFL). Moreover, the engraftment of distal organoids (MS5_CTRL vs. MS5_FS12) together with the comparison of local (membrane-bound) vs. systemic (soluble) delivery of human FLT3L in vivo, supported the notion 
Fig. 6 The MS5_FS12 niche supports human DC development in vivo. a Flow cytometry of Matrigel organoids containing either MS5_CTRL or MS5_FS12. Graphs show frequency of $\mathrm{cDC1}$ and $\mathrm{cDC} 2$ within $\mathrm{CD} 45^{+}$cells $\left(n=14\right.$ donors in 6 independent experiments). ${ }^{\star \star} p<0.01{ }^{\star \star \star \star} p<0.0001$, two-tailed paired Student's t-test. b Immunofluorescence of MS5_FS12 organoids sections stained for huCD45+ (green) and Clec9A+ (red) or and CD1c ${ }^{+}$(red). Nuclei stained with Hoechst (blue). Scale bar $=50 \mu \mathrm{m}(n=2$ Matrigel organoids). c Left: Flow cytometry and quantification of CD123+CD303/4+ cells in MS5-CTRL and MS5_FS12 organoids ( $n=14$ donors in 6 independent experiments. Two-tailed paired Student's t-test). Middle: Gating strategy discriminating $\mathrm{AXL}^{-} \mathrm{CD} 327 \mathrm{lo} /-\mathrm{pDC}$ and $\mathrm{AXL}+\mathrm{CD} 327^{+}$pre/AS-DC within CD123+CD45RA ${ }^{+}$cells in MS5-FS12 organoids. Bar graph illustrates frequency of each subset within $\mathrm{CD} 123^{+} \mathrm{CD} 45 \mathrm{RA}+$ cells $(n=4$ donors). Right: Frequency of pre/AS-DC within CD45+ cells in MS5_CTRL vs. MS5_FS12 organoids ( $n=7$ donors in 4 independent experiments). ${ }^{*} p<0.05$, two-tailed paired Student's $t$-test. d GSEA of pDC and pre/AS-DC using published gene signatures ${ }^{20}$. Statistical significance defined by the FDR $q$-value calculated by GSEA software (www.broad.mit.edu/gsea) using default parameters. e CyTOF analysis comparing CD45+HLA-DR ${ }^{+}$cells in MS5_FS12 and MS5-CTRL organoids. Pie charts display frequency within CD45+HLA-DR ${ }^{+}$cells (mean of $n=2$ donors in 2 independent experiments). f Frequency of $c D C 1, c D C 2, p D C$, pre/AS-DC and total CD123+CD45RA ${ }^{+}$cells recovered from physically separated plugs containing either MS5_CTRL or MS5_FS12 in the same recipient $\left(n=3\right.$ donors in one experiment). ${ }^{*} p<0.05$, two-tailed paired Student's $t$-test. $\mathbf{g}$ NSG mice injected subcutaneously either with MS5_CTRL or MS5_FS12 stromal cells. Human recombinant FLT3L administered intraperitoneum to mice bearing MS5_CTRL plugs (10 $\mu \mathrm{g} /$ mouse/injection) (MS5_CTRL+recFL). Frequency of human cDC1, cDC2, and pDC in subcutaneous organoids (left) and murine $\mathrm{CDC1}, \mathrm{CDC} 2$ and pDC in the spleen (center) were reported. Circulating recombinant FLT3L levels were measured by ELISA (right). $n=4$ mice/group in 2 independent experiments. ${ }^{\star} p<0.05,{ }^{\star \star} p<0.01$, one-way ANOVA test with Dunnett's T3 multiple comparisons. $\mathbf{h}$ Frequency of differentiated subsets within huCD45+ cells in MS5_FS12 plugs at day 12. The number of biological replicates ( $n$ ) is reported. Data presented as floating bars ranging from min to max and line represents median $(\mathbf{a}, \mathbf{c}, \mathbf{f}, \mathbf{h})$ or as bar graphs with mean $\pm \mathrm{SEM}(\mathbf{c}, \mathbf{g})$.

that cell-associated FLT3L delivered by engineered stromal cells significantly improves the development of human DCs in NSG mice.

Several protocols have been proposed for the in vitro differentiation of human cDCs from CD34 ${ }^{+} \mathrm{HSPCs}^{7,37-39,46,47}$. In vitro-differentiated $\mathrm{CDC} 1 \mathrm{~s}$ have been shown to fully recapitulate both the phenotype and function of circulating bona fide blood $\mathrm{cDC} 1 \mathrm{~s}^{8,38,46,47}$ including high levels of IRF8 expression and Batf3-dependency in vitro ${ }^{13}$, as well as IRF8-dependancy both in vivo ${ }^{67}$ and in vitro ${ }^{46}$. Conversely, several aspects have limited an exhaustive validation of in vitro generated $\mathrm{CD} 1 \mathrm{c}^{+} \mathrm{cDC} 2$-like cells such as the expression of $\mathrm{CD} 14^{7}$, the transcriptional alignment with monocyte-derived $\mathrm{DCs}^{38}$ or the lack of a highdimensional phenotypic characterization ${ }^{46,47}$.

Here we described a system (MS5_FS12), which efficiently supports the differentiation of both $\mathrm{CD} 141^{+} \mathrm{Clec} 9 \mathrm{~A}^{+} \mathrm{cDC} 1 \mathrm{~s}$ and CD14 ${ }^{-} \mathrm{CD} 1 \mathrm{c}^{+}$cDC2s. However, cDC2s generated in MS5_FS12 cultures only partially recapitulate the phenotype of circulating blood cDC2s, as suggested by the lack of expression of $\mathrm{cDC} 2-$ specific markers such as FceRIa and CD5. Nevertheless, engineered MSCs display the unique advantage of being suitable for in vivo applications.

Immunodeficient mice provide a unique system to model the onset of human immune responses in realistic settings ${ }^{68}$. However, a reliable method to achieve the differentiation of human DCs in vivo has not been described, yet. Current protocols rely on the engraftment of human $\mathrm{CD} 34^{+}$HSPCs in sub-lethally irradiated immunodeficient mice (humanized mice). This strategy has not been successful in the generation of well-characterized circulating DC subsets ${ }^{68}$. Administration of supraphysiological levels of recombinant FLT3L has been shown to stimulate CDC differentiation upon reconstitution of $\mathrm{NSG}^{28,48}$ or $\mathrm{Flt3}^{-1-}$ BRGS $^{49}$ mice with human CD34 ${ }^{+}$HSPCs. However, the phenotype of $\mathrm{CD}_{141}{ }^{+} \mathrm{cDC} 1 \mathrm{~s}$ and $\mathrm{CD} 1 \mathrm{c}^{+} \mathrm{cDC} 2 \mathrm{~s}$ was poorly characterized and, despite exceptions ${ }^{50}$, tissue DCs were not generally investigated. These aspects represent an important limitation by precluding, for instance, the modeling of skin vaccination. Alternatively, transgenic mice expressing human GM-CSF and IL-3 (in the presence or absence of human SCF), either constitutively 69,70 or by replacing their murine counterparts $(\text { knock-in })^{71}$, have been generated. Despite displaying higher levels of myeloid reconstitution, as well as the presence of human alveolar macrophages in the lungs of humanized mice ${ }^{71}$, this approach did not improve the development of human $\mathrm{cDCs}$ in lymphoid and non-lymphoid tissues of engrafted animals.
We demonstrated that MS5_FS12 support the differentiation of human $\mathrm{cDCs}$ in vivo in subcutaneous organoids in NSG mice. High-dimensional mass cytometry (Cytof) and transcriptomic (RNA-seq) analysis of in vivo generated cells confirmed their phenotypic and transcriptional alignment to circulating blood $\mathrm{cDC} 1 \mathrm{~s}$ and $\mathrm{cDC} 2 \mathrm{~s}$. More importantly, $\mathrm{cDC} 2 \mathrm{~s}$ generated in vivo better resemble their physiologically circulating counterparts by expressing higher levels of FceRIa, CD172a, CD5, CD2, and BTLA when compared to in vitro differentiated cells. The lower expression of activation markers such as CD86, CD80 and MHC molecules also suggests that in vivo $\mathrm{CDC} 2 \mathrm{~s}$ displayed a less mature phenotype than in vitro-generated cells.

Moreover, MS5_FS12 niche was capable of supporting the local maintenance and expansion of human HSPCs as well as pre/ASDCs, resulting in the persistence of a long-lasting source of progenitors capable of undergoing DC differentiation. To our knowledge, this is the first time that a well-characterized system supporting the development of human pre/AS-DC is reported.

Collectively, we have demonstrated that the engineered stromal cells MS5_FS12 give rise to a synthetic hematopoietic niche when injected subcutaneously in NSG mice. The niche microenvironment efficiently supports the expansion of CD34 ${ }^{+}$HSPCs and human DCs subsets ( $\mathrm{CDC} 1, \mathrm{cDC} 2$, and pre/AS-DC) can be detected as early as day 12 in a radiation-free environment. Importantly, in vitro culture system imposes a certain level of spontaneous activation that is not found in primary circulating blood DCs. Differentiation of human cDCs within humanized mice limit this phenomenon to a level closer to the maturation state of circulating primary cDCs. Hence, this approach represents a versatile system to study human DC development and function in vivo.

\section{Methods}

Mice. All in vivo experiments were performed using NOD.Cg-Prdc scid $I l 2 r g^{t m 1} W_{j l}$ SzJ (NSG) mice (JAX \#005557). All mice were used between 8 and 12 weeks of age. They were maintained in specific-pathogen-free conditions and handled according to protocols approved by the UK Home Office.

Generation of engineered MSCs. Human FLT3L, SCF, and TPO were amplified by PCR from cDNA expression plasmids (Origene) and cloned into pMX retrovira vectors (vectors details in Supplementary Table 1). Lentiviral vector pBABE-puroSDF-1 alpha was a gift from Bob Weinberg (Addgene plasmid \#12270) ${ }^{72}$. Viral particles were generated using the retroviral packaging plasmid pCL-Ampho and a second generation lentiviral packaging system (psPAX2 and pMD2.G), respectively. MS5 cells were first transduced with CXCL12 lentiviral vector and selected using $15 \mu \mathrm{g} / \mathrm{ml}$ of Puromycin (Thermo Fisher). Then, a combination of single or multiple cytokines were used to transduce MS5 cells as illustrated in 
a
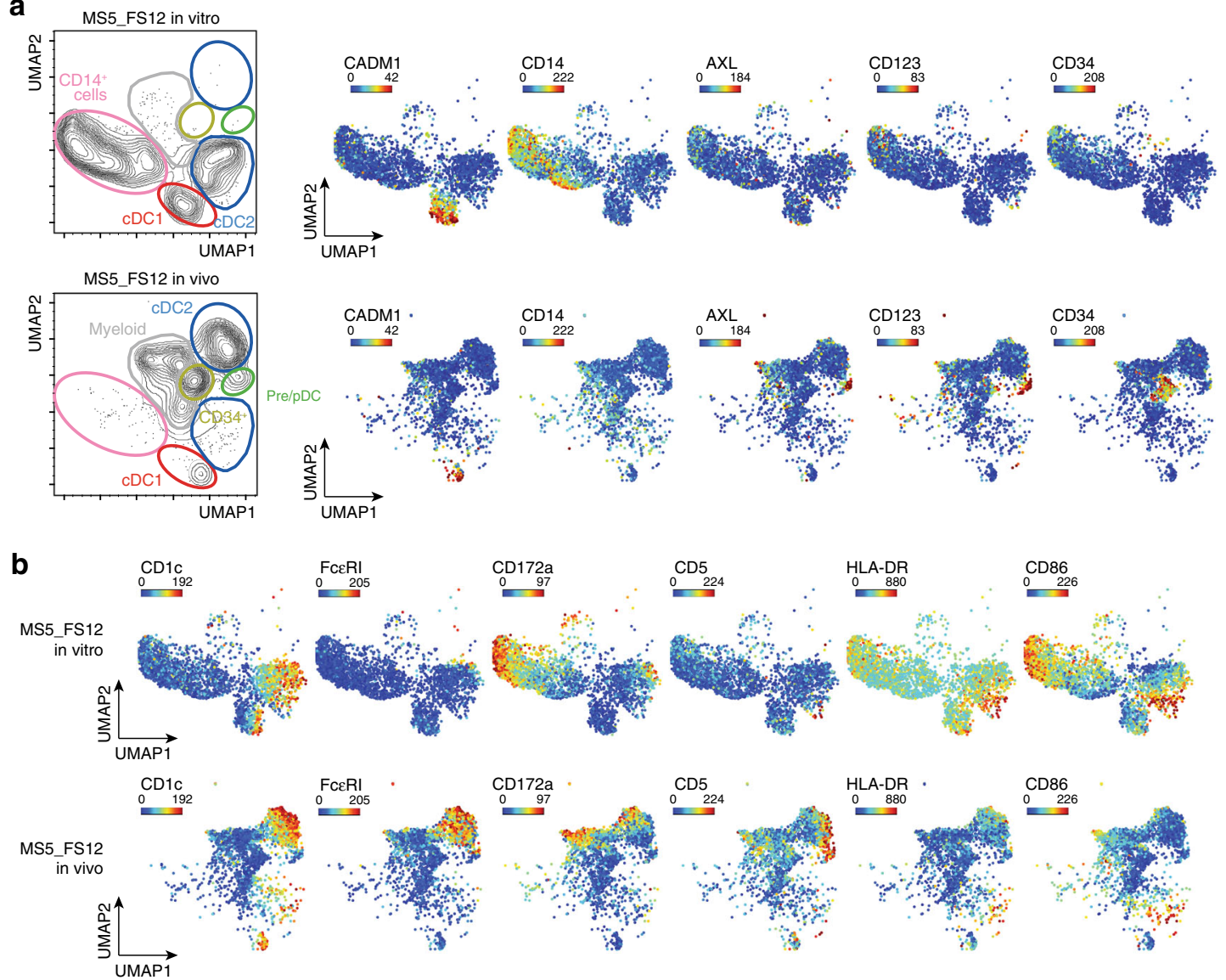

C

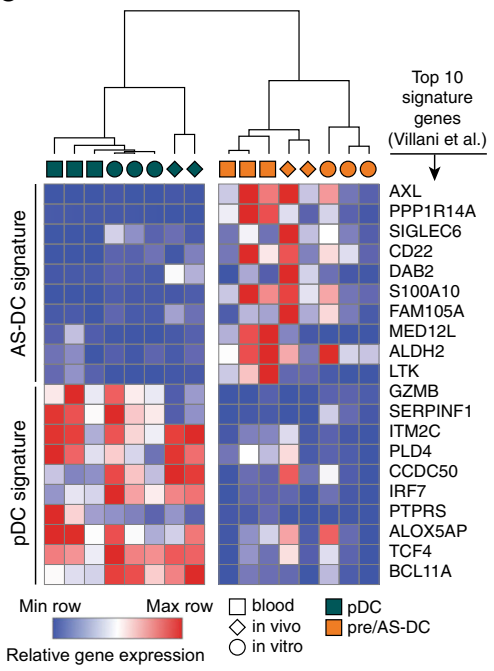

d

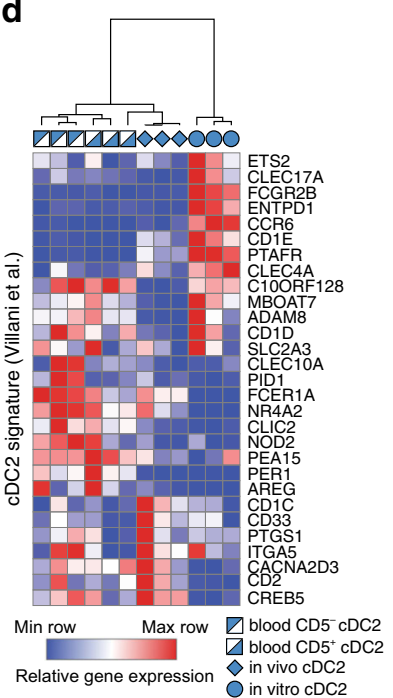

e

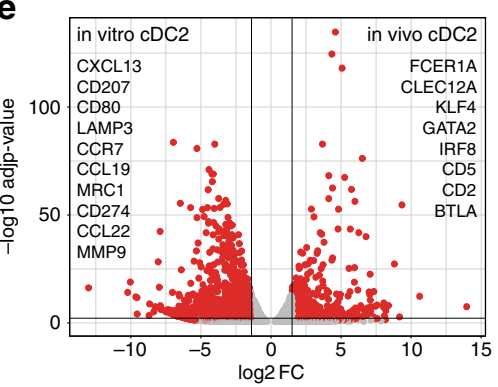

f

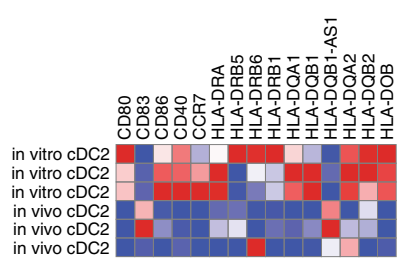

Fig. 7 cDC2 generated in vivo faithfully align to blood cDC2. a UMAP plots of CyTOF data comparing CD45+HLA-DR ${ }^{+}$cells generated using MS5_FS12 stromal cells either in vitro or in vivo. Relative expression of selected markers is shown for each condition. $\mathbf{b}$ Relative expression of selected markers highlighting the phenotypic differences between cDC2s generated in vitro and in vivo using MS5_FS12 stromal cells. c Heatmap displaying gene expression of the top ten genes of blood pDC and AS-DC published signatures 20 in pDC and pre/AS-DC generated in vitro, in vivo, and isolated from blood PBMC ( $n=2$ independent donors for pDC and pre/AS-DC generated in vivo and $n=3$ independent donors for $\mathrm{pDC}$ and pre/AS-DC generated in vitro or isolated from peripheral blood). d Heatmap displaying gene expression of the blood cDC2 published signature 20 in cDC2 cells generated in vitro, in vivo and isolated from blood PBMC ( $n=3$ independent donors). e Volcano plot showing differentially expressed genes between in vitro and in vivo generated cDC2 (Log2FC > 1.5, adjusted $p$-value $<0.05$ ). Statistical significance was calculated using Wald test with a Benjamin-Hochberg $p$-value correction ( $n=3$ independent donors per group). $\mathbf{f}$ Heatmap displaying gene expression of activation markers and co-stimulatory molecules expressed in $\mathrm{CDC} 2$ generated in vivo and in vitro ( $n=3$ independent donors). 
a

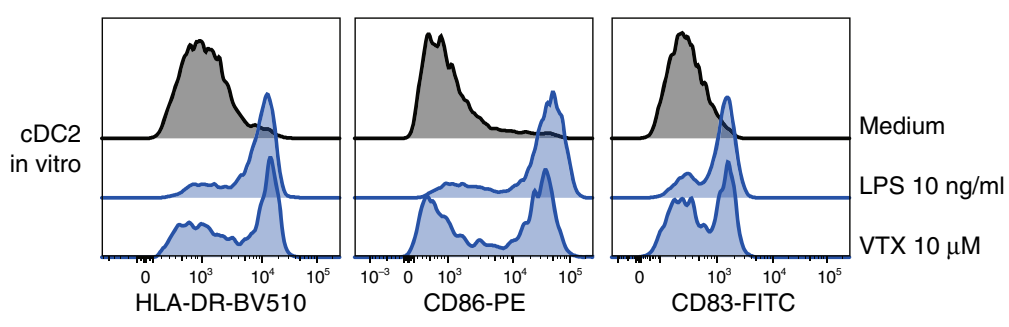

b
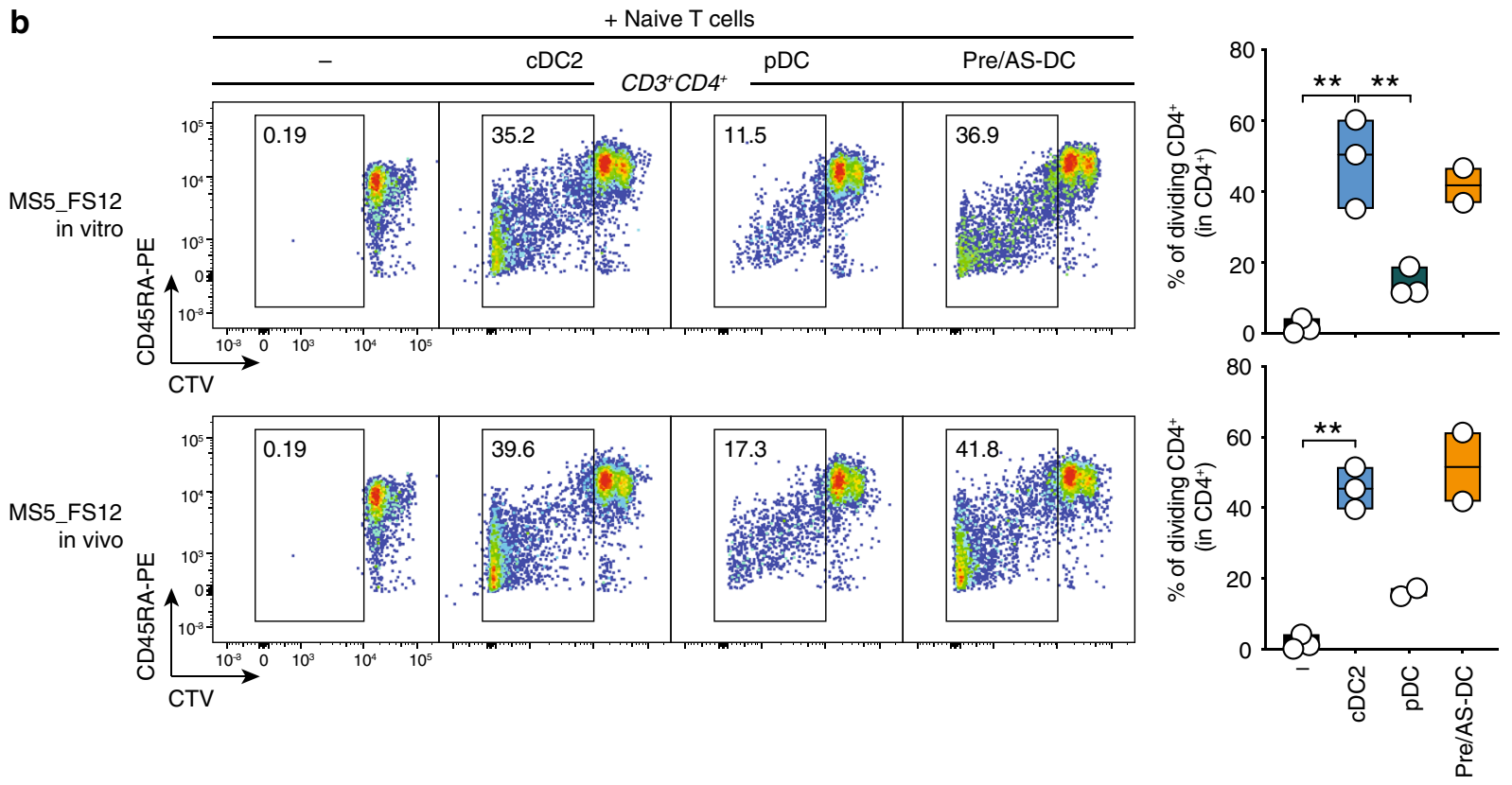

C
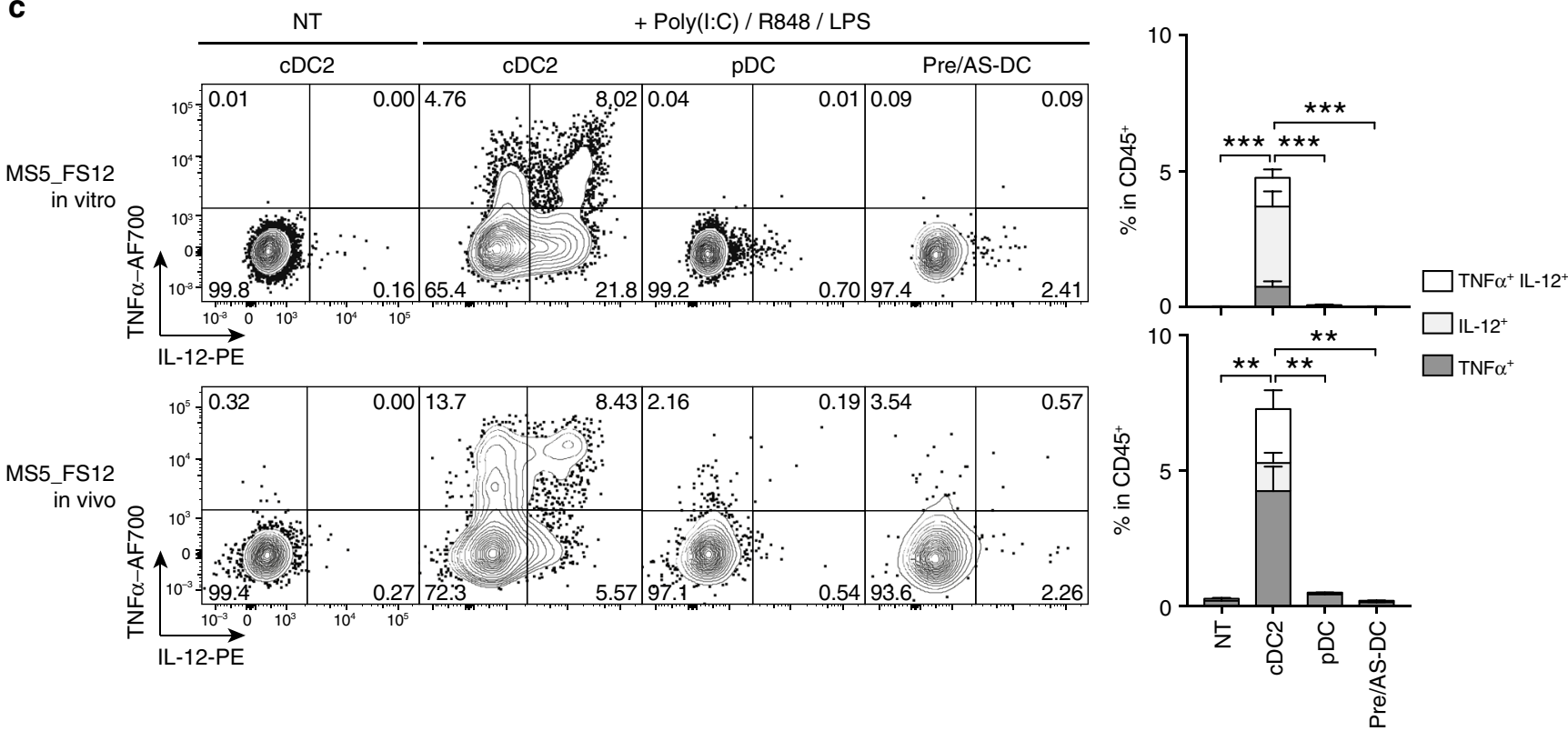

Supplementary Fig. 2a. Cells expressing human membrane-bound FLT3L and SCF were sorted according to antibody staining of the transmembrane proteins (antibodies listed in Supplementary Table 3). TPO-expressing cells were sorted according to the expression of mCherry reporter.

Flow cytometry and fluorescent-activated cell sorting. Extracellular staining of cells was preformed in FACS buffer, consisting in phosphate-buffered saline (PBS) (Life Technologies), 1\% bovine serum albumin (BSA) (Apollo Scientific) and $2 \mathrm{mM}$ EDTA (Life Technologies). For intracellular staining, samples were fixed and permeabilized using the Cytofix/Cytoperm ${ }^{\mathrm{TM}}$ kit (BD Biosciences) according to manufacturers' instructions. Antibodies used in all experiments are listed in Supplementary Table 3. Flow cytometry analysis was performed on LSR Fortessa II (BD Biosciences, BD FACSDiva Software) and data were analyzed using FlowJo software (TreeStar, version 10.2). Cell sorting was performed using AriaII (BD Biosciences, BD FACSDiva Software).

Cell lines maintenance and primary cells isolation. MS5 $5^{51}$ and eMSC lines were cultured in IMDM (Life Technologies) supplemented with $10 \%$ heat-inactivated fetal bovine serum (FBS) (Life Technologies), penicillin/streptomycin (Life Technologies), $50 \mu \mathrm{M} \beta$-mercaptoethanol (Life Technologies), and maintained at $37^{\circ} \mathrm{C}$ 
Fig. 8 In vitro and in vivo cDC2 functionally align to blood cDC2. a Histograms showing upregulation of activation markers HLA-DR, CD86 and CD83 in in vitro-differentiated cDC2 in response to TLR4 (LPS) and TLR8 (VTX-2337) overnight (16 h) stimulation. b Representative FACS plots and quantification of mixed lymphocyte reaction (MLR) using in vitro and in vivo differentiated cDC2, pDC, and pre/AS-DC. FACS-sorted DC subsets were activated overnight (16 h) using a TLR agonist cocktail (LPS $10 \mathrm{ng} / \mathrm{ml}$, R848 $1 \mu \mathrm{g} / \mathrm{ml}$ and Poly(I:C) $25 \mu \mathrm{g} / \mathrm{ml}$ ) and co-cultured with CTV-labeled naive T cells for 7 days ( $n=2$ independent donors for pDC generated in vivo and pre/AS-DC generated in vitro and in vivo and $n=3$ independent donors for in vitro and in vivo generated $\mathrm{CDC} 2$ and in vitro generated $\mathrm{pDC}$ in two independent experiments). ${ }^{\star \star} p<0.01$, one-way ANOVA test with Holm-Sidak's multiple comparisons. c Intracellular flow cytometry analysis of TNF $\alpha$ and IL-12 expression in in vitro and in vivo-differentiated DC subsets upon overnight (16 $\mathrm{h}$ ) stimulation with TLR agonist cocktail (LPS $10 \mathrm{ng} / \mathrm{ml}, \mathrm{R} 8481 \mu \mathrm{g} / \mathrm{ml}$, and Poly (I:C) $25 \mu \mathrm{g} / \mathrm{ml}$ ) as compared to unstimulated cells (NT). $n=4$ independent cord blood donors. ${ }^{\star \star} p<0.01,{ }^{\star \star \star} p<0.001$, one-way ANOVA test Holm-Sidak's multiple comparisons. Data are presented as floating bars ranging from min to max and line represents median $\mathbf{b}$ or as bar graphs with mean \pm SEM (c).

$5 \% \mathrm{CO}_{2}$. OP9 and OP9_FLT3L were cultured in complete $\alpha$-MEM (Life Technologies) supplemented with $20 \%$ not heat-inactivated FBS, penicillin/streptomycin, $50 \mu \mathrm{M} \beta$-mercaptoethanol and maintained at $37^{\circ} \mathrm{C} 5 \% \mathrm{CO}_{2}$.

Cord blood units were obtained from Anthony Nolan Cell Therapy Centre (ANCTC). Peripheral blood mononuclear cells (PBMCs) were isolated by gradient centrifugation using Ficoll-Paque (GE Healthcare) and $\mathrm{CD}_{3}{ }^{+}$hematopoietic progenitors were isolated using CD34 MicroBead Kit UltraPure (Miltenyi Biotec).

Adult peripheral blood was obtained from healthy volunteers from NHS Blood and Transplant. PBMCs were isolated by Ficoll-Paque gradient centrifugation. Cells were collected in FACS buffer and used for downstream applications.

Human dendritic cell differentiation in vitro. For in vitro differentiation of human DCs, MS5, OP9 or eMSCs were seeded in a 96-well plate (flat bottom) at a density of $10^{4}$ cells/well. The following day, $10^{4}$ cord blood-derived CD34+ cells/ well were seeded on top of stromal cells in complete IMDM $(10 \%$ heat-inactivated FBS, penicillin/streptomycin, $50 \mu \mathrm{M} \beta$-mercaptoethanol) and maintained at $37^{\circ} \mathrm{C}$ $5 \% \mathrm{CO} 2$. Half of the medium was replaced at day 5 and 10 , and cells were collected with a solution of PBS $5 \mathrm{mM}$ EDTA (at $4{ }^{\circ} \mathrm{C}$ ) at day 15 for flow cytometry analysis. For recombinant FLT3L experiments, human FLT3L (Celldex) $100 \mathrm{ng} / \mathrm{ml}$ was used. For transwell experiments, 24 -well plates with $0.4 \mu \mathrm{m}$ pores Transwell ${ }^{\circledR}$ inserts (Corning) were used. Stromal cells were plated at a density of $10^{5}$ cells/well and $7 \times$ $10^{4}$ cord blood-derived CD34 ${ }^{+}$progenitors were added the following day in each well. Half of the medium was replaced in both the top and bottom well at day 5 and 10. For GM-CSF blocking experiments, $2 \mu \mathrm{g} / \mathrm{ml}$ human GM-CSF neutralizing antibody (R\&D catalog number \#AF-215-SP) and isotype control (R\&D catalog number \#AB-108-C) were added to the culture medium. The presence of human GM-CSF in the supernatant of eMSCs co-cultures with CD $34^{+}$HSPCs was assessed by enzyme-linked immunosorbent assay (ELISA) using the human GMCSF ELISA MAX kit (Biolegend) as per manufacturer's instructions.

RNA-seq and data processing. RNA-seq analysis was performed in two independent experiments. In the first experiment, human $\mathrm{cDC} 1 \mathrm{~s}$ and $\mathrm{cDC} 2 \mathrm{~s}$ from peripheral blood of $n=3$ healthy individuals and in vitro generated CD141 ${ }^{+} \mathrm{Clec} 9 \mathrm{~A}^{+}, \mathrm{CD} 14^{-} \mathrm{CD} 1 \mathrm{c}^{+} \mathrm{CD} 206^{-}$, and $\mathrm{CD} 14^{-} \mathrm{CD} 1 \mathrm{c}^{+} \mathrm{CD} 206^{+}$cells from $n=3$ independent cord blood donors were FACS sorted. Up to 100 cells/subset were collected in lysis buffer (Takara Clontech) containing RNAse inhibitors. RNA-seq libraries were prepared using Labcyte Echo 525 contactless liquid handling system (Labcyte, Inc.). In brief, ERCC mix (Thermo Fisher) was added to each sample and first strand full-length cDNA was generated with a modified protocol of the SMARTseq v4 Ultra Low Input RNA Kit (Takara Clontech) using poly dT primers and a template switching oligo. Full-length cDNA was amplified using SeqAmp ${ }^{\text {tx }}$ DNA Polymerase (Takara Clontech). 12 ng of amplified cDNA from each sample was used to generate non-stranded RNA libraries using a modified protocol of the Ovation Ultralow System V2 1-96 kit (NuGEN). In brief, amplified cDNA was fragmented through sonication on Covaris E220 (Covaris, Inc.), repaired and polished followed by ligation of indexed adapters. Adapter ligated cDNA were pooled before final amplification to add flow cell primers. Libraries were sequenced on HiSeq2500 (Illumina Cambridge) for 100 cycles PE in Rapid mode. The raw sequencing data was initially processed using open source, web-based platform Galaxy (version 18.05.rc1) (https://usegalaxy.org). Reads were filtered for quality with more than $80 \%$ of the sequence having quality score $>33$ using FastQC tool. Mapping against reference genome was performed with Hisat2 to the hg38 human genome. Adapter sequences were detected automatically with TrimGalore!. Reads under $20 \mathrm{bp}$ were discarded. All processed sequencing files were imported in Partek $^{\circledast}$ Flow $^{\circledast}$ software (Partek, Inc., build 7.0.18.0514) and the gene count data was normalized using FPKM.

In the second experiment, human $\mathrm{CD}^{-}{ }^{-} \mathrm{cDC} 2, \mathrm{CD}^{+}+\mathrm{cDC} 2, \mathrm{pDC}$, and pre/ASDC from peripheral blood of $n=3$ healthy individuals and $\mathrm{CDC} 2, \mathrm{pDC}$ and pre/ AS-DC generated both in vivo and in vitro from $n=2 / 3$ independent cord blood donors were FACS sorted. Between 100 and 1000 cells/subset were collected in TRIzol ${ }^{\circledast}$ (Thermo Fisher) and stored at $-80^{\circ} \mathrm{C}$. Frozen samples were shipped to GENEWIZ $^{\circledR}$ where they were processed. RNA was extracted and libraries were prepared using an ultralow input RNA library preparation kit (Illumina). Libraries were sequenced on HiSeq2500 (Illumina)
The raw sequencing data was initially aligned on the human reference genome hg38 using STAR aligner (v2.5.3a $)^{73}$ (parameters: --outFilterType BySJout -outFilterMultimapNmax 20 --alignSJoverhangMin 8

--alignSJDBoverhangMin 1 --

outFilterMismatchNmax 999 --outFilterMismatchNoerLmax 0.04

--alignIntronMin 20 --

alignIntronMax 1000000 --alignMatesGapMax 1000000 --outSAMprimaryFlag OneBestScore --outMultimapperOrder Random --outSAMattributes All). Raw read counts matrix was also made with STAR (using the parameter --quantMode GeneCounts).

RNA-sequencing analysis. The average gene expression of $n=3$ blood donors for cDC1s, $n=2$ blood donors for $\mathrm{cDC} 2$ and $n=3$ cord blood units for in vitro generated subsets were used for RNA-seq data analysis.

Hierarchical clustering was performed in Morpheus (Broad Institute, https:// software.broadinstitute.org/morpheus/) using one minus Pearson's correlation and average linkage.

GSEA (www.broad.mit.edu/gsea) ${ }^{58}$ was used to assess the expression of gene signatures specific for blood $\mathrm{cDC} 1, \mathrm{cDC} 2, \mathrm{DC} 3, \mathrm{pDC}$, and $\mathrm{AS}-\mathrm{DC}$ in in vitro and in vivo generated subsets. To simultaneously visualize pairwise comparisons of transcriptomes from cord blood-derived cDCs, the BubbleMap module of BubbleGum ${ }^{59}$ was used. Results were considered significant when the $p$-value was below 0.05 and the FDR $(q)$ value was below 0.25 . The GSEA was performed using previously published gene signatures defining blood $\mathrm{cDC} 1, \mathrm{cDC} 2, \mathrm{DC} 3, \mathrm{pDC}$, and AS-DC ${ }^{20}$, as well as newly generated signatures using the GeneSign module of BubbleMap ${ }^{59}$ (Supplementary Table 6). Briefly, the transciptome of blood cDC1s and blood $\mathrm{CD} \mathrm{c}^{+}$cells was taken from previously published datasets ${ }^{60}$.

$\mathrm{cDC} 1>\mathrm{CD} 1 \mathrm{c}^{+}$and $\mathrm{CD} 1 \mathrm{c}^{+}>\mathrm{CDC} 1$ signatures were defined using the "Min(test) vs. $\operatorname{Max}(\mathrm{ref})$ " statistical method with a minimal linear fold change $=2$ and a maximal $\mathrm{FDR}=0.01$.

Heatmaps displaying the expression pattern of gene signatures for $\mathrm{CDC1}, \mathrm{CD} 1 \mathrm{c}$ + cells, pDC, AS-DC, and cDC2 were generated using Morpheus (Broad Institute, https://software.broadinstitute.org/morpheus/).

The 2000 genes defining the "in vitro culture imprinting" were identified using Morpheus as the mean difference of expression values between two groups: the in vitro generated cells (including cDC1, cDC2 CD206 ${ }^{-}$and $\mathrm{cDC} 2 \mathrm{CD} 206^{+}$) versus ex vivo-isolated subsets (blood $\mathrm{cDC} 1$ and blood $\mathrm{cDC} 2$ ).

The 2872 genes defining the "in vivo signature" were identified by DEG analysis using the R package DESeq2 (version 1.24.0) ${ }^{74}$ with a Benjamin-Hochberg $p$-value correction $^{75}(\log 2 \mathrm{FC}>1.5$, adjusted $p$-value $<0.01)$. The volcano plot displaying the differentially expressed genes between in vivo and in vitro differentiated $\mathrm{CDC} 2$ was generated using $\mathrm{R}$ package ggplot2 (version 3.2.1) ${ }^{76}(\log 2 \mathrm{FC}>1.5$, adjusted $p$-value $<0.05)$. All the analysis from the raw counts matrix were performed in Rstudio (1.2.5001) using the version 3.6.1 of R. Pathway analysis was performed using ConsensusPathDB (cpdb.molgen.mpg.de) ${ }^{77}$ and WikiPathways database.

Mass cytometry acquisition and data analysis. For mass cytometry, preconjugated or purified antibodies were obtained from Invitrogen, Fluidigm (preconjugated antibodies), Biolegend, eBioscience, Becton Dickinson, or R\&D Systems as listed in Supplementary Table 5. For some markers, fluorophore- or biotinconjugated antibodies were used as primary antibodies, followed by secondary labeling with anti-fluorophore metal-conjugated antibodies (such as the anti-FITC clone FIT-22) or metal-conjugated streptavidin, produced as previously described $^{78}$. Briefly, $3 \times 10^{6}$ cells/well in a U-bottom 96-well plate (BD Falcon) were washed once with $200 \mu \mathrm{L}$ FACS buffer (4\% FBS, 2 mM EDTA, 0.05\% Azide in $1 \times$ PBS) and then stained with $100 \mu \mathrm{L} 200 \mu \mathrm{M}$ cisplatin (Sigma-Aldrich) for $5 \mathrm{~min}$ on ice to exclude dead cells. Cells were then incubated with anti-CADM1-biotin antibody in a $50 \mu \mathrm{L}$ reaction for $30 \mathrm{~min}$ on ice. Cells were washed twice with FACS buffer and incubated with $50 \mu \mathrm{L}$ heavy-metal isotope-conjugated secondary $\mathrm{mAb}$ cocktail for $30 \mathrm{~min}$ on ice. Cells were then washed twice with FACS buffer and once with PBS before fixation with $200 \mu \mathrm{L} 2 \%$ paraformaldehyde (PFA; Electron Microscopy Sciences) in PBS overnight or longer. Following fixation, the cells were pelleted and resuspended in $200 \mu \mathrm{L} 1 \mathrm{X}$ permeabilization buffer (Biolegend) for 5 minutes at room temperature to enable intracellular labeling. Cells were then 
incubated with metal-conjugated anti-CD68 in a $50 \mu \mathrm{L}$ reaction for $30 \mathrm{~min}$ on ice. Finally, the cells were washed once with permeabilization buffer and then with PBS before barcoding.

Bromoacetamidobenzyl-EDTA (BABE)-linked metal barcodes were prepared by dissolving BABE (Dojindo) in $100 \mathrm{mM}$ HEPES buffer (Gibco) to a final concentration of $2 \mathrm{mM}$. Isotopically-purified $\mathrm{PdCl}_{2}$ (Trace Sciences Inc.) was then added to the $2 \mathrm{mM}$ BABE solution to a final concentration of $0.5 \mathrm{mM}$. Similarly, DOTA-maleimide (DM)-linked metal barcodes were prepared by dissolving DM (Macrocyclics) in L buffer (MAXPAR) to a final concentration of $1 \mathrm{mM}$. $\mathrm{RhCl}_{3}$ (Sigma) and isotopically-purified $\mathrm{LnCl}_{3}$ was then added to the $\mathrm{DM}$ solution at $0.5 \mathrm{mM}$ final concentration. Six metal barcodes were used: BABE-Pd-102, BABEPd-104, BABE-Pd-106, BABE-Pd-108, BABE-Pd-110, and DM-Ln-113.

All BABE and DM-metal solution mixtures were immediately snap-frozen in liquid nitrogen and stored at $-80^{\circ} \mathrm{C}$. A unique dual combination of barcodes was chosen to stain each sample. Barcode Pd-102 was used at 1:4000 dilution, P d-104 at 1:2000, Pd-106 and Pd-108 at 1:1000, Pd-110 and Ln-113 at 1:500. Cells were incubated with $100 \mu \mathrm{L}$ barcode in PBS for $30 \mathrm{~min}$ on ice, washed in permeabilization buffer and then incubated in FACS buffer for $10 \mathrm{~min}$ on ice. Cells were then pelleted and resuspended in $100 \mu \mathrm{L}$ nucleic acid Ir-Intercalator (MAXPAR) in 2\% PFA/PBS (1:2000), at room temperature. After $20 \mathrm{~min}$, cells were washed twice with FACS buffer and twice with water before a final resuspension in water. In each set, the cells were pooled from all samples, counted, and diluted to $0.5 \times 10^{6}$ cells $/ \mathrm{mL}$. EQ Four Element Calibration Beads (DVS Science, Fluidigm) were added at a $1 \%$ concentration prior to acquisition. Cell data were acquired and analyzed using a CyTOF Mass cytometer (Fluidigm).

The CyTOF data were exported in a conventional flow cytometry file (.fcs) format and normalized using previously described software ${ }^{79}$. Events with zero values were randomly assigned a value between 0 and -1 using a custom $\mathrm{R}$ script employed in a previous version of mass cytometry software ${ }^{80}$. Cells for each barcode were deconvolved using the Boolean gating algorithm within FlowJo. The $\mathrm{CD}^{2} 5^{+} \mathrm{Lin}(\mathrm{CD} 7 / \mathrm{CD} 14 / \mathrm{CD} 15 / \mathrm{CD} 16 / \mathrm{CD} 19 / \mathrm{CD} 34)^{-} \mathrm{HLA}^{-\mathrm{DR}^{+}}$population of PBMC were gated using FlowJo and exported as an.fcs file. Marker expression values were transformed using the logicle transformation function ${ }^{81}$. Random subsampling without replacement was performed to select 20,000 cell events.

The transformed values of sub-sampled cell events were then subjected to UMAP dimension reduction 61,82 using all markers. We used the 2.4 .0 release of UMAP, implemented in Python, but executed through the reticulate $\mathrm{R}$ package to interface R objects with Python. For both mass-cytometry datasets we used UMAP using 15 nearest neighbors $(n n)$, a min_dist of 0.2 and euclidean distance.

Heatmaps displaying mean intensity values of CyTOF data were generated using Morpheus (Broad Institute, https://software.broadinstitute.org/morpheus/).

Human dendritic cell differentiation in vivo. Human cord blood-derived CD $34^{+}$ hematopoietic cells $\left(5-15 \times 10^{4}\right.$ cells/plug) were injected subcutaneously along with engineered stromal cells ( $1: 1$ to $1: 5$ ratio HSPC/MS5) in $200 \mu \mathrm{l}$ of ice-cold Matrigel $^{\circledR}$ (BD Biosciences). Mice were killed at day 12 of differentiation by cervical dislocation and Matrigel ${ }^{\circledR}$ plugs were collected. Subcutaneous Matrigel ${ }^{\circledast}$ plugs were recovered, cut in pieces and incubated in HBSS (Life Technologies) 1\% FBS, $0.37 \mathrm{U} / \mathrm{ml}$ Collagenase D (Roche), $10 \mu \mathrm{g} / \mathrm{ml}$ DNaseI (Roche), and $1 \mathrm{mg} / \mathrm{ml}$ Dispase (Sigma-Aldrich) for 30 minutes at $37^{\circ} \mathrm{C}$. After digestion, plugs were smashed on a $100 \mu \mathrm{m}$ strainer (Corning) and cells were collected and resuspended in FACS buffer for flow cytometry analysis.

Histology. Matrigel plugs were fixed with 1\% PFA (Alfa Aesar) for $1 \mathrm{hr}$ at $4{ }^{\circ} \mathrm{C}$, washed and incubated in 34\% sucrose solution (Sigma-Aldrich) overnight at $4{ }^{\circ} \mathrm{C}$. Plugs were embedded in Cryomatrix (Thermo Fisher) and frozen for cryostat sectioning $(9 \mu \mathrm{m}$-thick). Sections were permeabilized using $0.5 \%$ saponin (SigmaAldrich), 2\% BSA (Sigma-Aldrich), 1\% FBS (Life Technologies) for $30 \mathrm{~min}$ at room temperature. For human DCs staining, plug sections were incubated with $1 \%$ rat anti-mouse CD16/32 (homemade) for 30 min to block unspecific binding sites. Sections were labeled overnight at $4{ }^{\circ} \mathrm{C}$ with mouse anti-human CD1c-PE (L161, Biolegend) or mouse anti-human Clec9A-PE (8F9, Biolegend) followed by incubation for $1 \mathrm{~h}$ at room temperature with goat anti-mouse Cy3 (Jackson laboratory). After extensive washes, sections were labeled with mouse anti-human CD45-APC (HI30, Biolegend) for $1 \mathrm{hr}$ at room temperature. For human $\mathrm{CD}_{3} 4^{+}$progenitors staining, plugs sections were labeled overnight with purified mouse anti-human CD45 (HI30, Biolegend) followed by $1 \mathrm{hr}$ incubation at room temperature with goat anti-mouse $\mathrm{Cy} 3$. After extensive washes, sections were labeled with mouse anti-human CD34- APC (561, Biolegend) for $1 \mathrm{~h}$ at room temperature. To detect murine endothelial cells, sections were labeled with purified rat anti-mouse CD31 (MEC13.3, Biolegend) and mouse anti-human CD45 (HI30, Biolegend) overnight followed by $1 \mathrm{~h}$ incubation at room temperature with goat anti-mouse $\mathrm{Cy} 3$ (Jackson laboratory) and goat anti-rat Cy5 (Jackson laboratory). All sections were labeled with Hoechst (Molecular Probes, Thermo Fisher) for nuclei staining 5 minutes at room temperature and mounted with Prolong diamond (Thermo Scientific). Slides were imaged using a SP5 (Leica Application Suite) and analyzed with Fiji software.
Mixed lymphocyte reaction. Cord blood-derived DC subsets differentiated in vitro and in vivo were FACS-sorted into a $\mathrm{V}$ bottom 96-well plate (Corning) $\left(10^{4}\right.$ cells/well $)$ and activated overnight $(16 \mathrm{~h})$ using a TLR agonists cocktail containing LPS $10 \mathrm{ng} / \mathrm{ml}, \mathrm{R} 8481 \mu \mathrm{g} / \mathrm{ml}$, and Poly(I:C) $25 \mu \mathrm{g} / \mathrm{ml}$.

PBMCs were isolated by gradient centrifugation using Ficoll-Paque (GE Healthcare) and labeled with Cell Trace Violet (Thermo Fisher) according to manufacturer's user guide. CTV-labeled T cells were then isolated using a Pan naive T-cell isolation kit (Miltenyi Biotec) according to manufacturer's instructions and isolation purity $(\geq 95 \%)$ was assessed by flow cytomery. Isolated naive $\mathrm{T}$ cells $\left(10^{5}\right.$ cells/well $)$ were seeded together with FACS-sorted DC (1:10 ratio DC/T cells) and incubated at $37^{\circ} \mathrm{C} 5 \% \mathrm{CO}_{2}$ for 7 days.

Statistical analysis. In all graphs each dot represents an independent cord blood donor and lines represent the median value. The number of biological as well as experimental replicates is indicated in figure legend. For each experiment, the appropriate statistical test is stated in figure legend. Statistical significance was defined as $P<0.05$. All graphs and statistical analysis was performed using GraphPad Prism.

Reporting summary. Further information on research design is available in the Nature Research Reporting Summary linked to this article.

\section{Data availability}

Data that support the findings of this study have been deposited in NCBI's Gene Expression Omnibus (GEO) and are accessible through GEO accession codes GSE144435 [https://www.ncbi.nlm.nih.gov/geo/query/acc.cgi?acc=GSE144435] and GSE145803 [https://www.ncbi.nlm.nih.gov/geo/query/acc.cgi?acc=GSE145803].

Received: 25 October 2018; Accepted: 18 March 2020;

Published online: 28 April 2020

\section{References}

1. Banchereau, J. \& Steinman, R. M. Dendritic cells and the control of immunity. Nature 392, 245-252 (1998)

2. Steinman, R. M., Hawiger, D. \& Nussenzweig, M. C. Tolerogenic dendritic cells. Annu Rev. Immunol. 21, 685-711 (2003).

3. Palucka, K. \& Banchereau, J. Dendritic-cell-based therapeutic cancer vaccines. Immunity 39, 38-48 (2013).

4. Bachem, A. et al. Superior antigen cross-presentation and XCR1 expression define human CD11c+CD141+ cells as homologues of mouse CD8+ dendritic cells. J. Exp. Med. 207, 1273-1281 (2010).

5. Crozat, $\mathrm{K}$. et al. The XC chemokine receptor 1 is a conserved selective marker of mammalian cells homologous to mouse CD8alpha+ dendritic cells. J. Exp. Med. 207, 1283-1292 (2010).

6. Jongbloed, S. L. et al. Human CD141+ (BDCA-3)+ dendritic cells (DCs) represent a unique myeloid DC subset that cross-presents necrotic cell antigens. J. Exp. Med. 207, 1247-1260 (2010).

7. Lee, J. et al. Restricted dendritic cell and monocyte progenitors in human cord blood and bone marrow. J. Exp. Med. 212, 385-399 (2015).

8. Helft, J. et al. Dendritic cell lineage potential in human early hematopoietic progenitors. Cell Rep. 20, 529-537 (2017).

9. Lee, J. et al. Lineage specification of human dendritic cells is marked by IRF8 expression in hematopoietic stem cells and multipotent progenitors. Nat. Immunol. 18, 877-888 (2017).

10. Breton, G. et al. Circulating precursors of human CD1c+ and CD141+ dendritic cells. J. Exp. Med. 212, 401-413 (2015).

11. Breton, G. et al. Human dendritic cells (DCs) are derived from distinct circulating precursors that are precommitted to become CD1c+ or CD141+ DCs. J. Exp. Med. 213, 2861-2870 (2016).

12. See, P. et al. Mapping the human DC lineage through the integration of highdimensional techniques. Science 356, pii: eaag3009 (2017).

13. Poulin, L. F. et al. DNGR-1 is a specific and universal marker of mouse and human Batf3-dependent dendritic cells in lymphoid and nonlymphoid tissues. Blood 119, 6052-6062 (2012).

14. Montoya, M. et al. Type I interferons produced by dendritic cells promote their phenotypic and functional activation. Blood 99, 3263-3271 (2002).

15. Haniffa, M. et al. Human tissues contain CD141hi cross-presenting dendritic cells with functional homology to mouse CD103+ nonlymphoid dendritic cells. Immunity 37, 60-73 (2012).

16. Schlitzer, A. et al. IRF4 transcription factor-dependent CD11b+ dendritic cells in human and mouse control mucosal $\mathrm{IL}^{-} 17$ cytokine responses. Immunity 38, 970-983 (2013)

17. Tamura, T. et al. IFN regulatory factor- 4 and -8 govern dendritic cell subset development and their functional diversity. J. Immunol. 174, 2573-2581 (2005). 
18. Segura, E. et al. Human inflammatory dendritic cells induce Th17 cell differentiation. Immunity 38, 336-348 (2013).

19. Segura, E. et al. Characterization of resident and migratory dendritic cells in human lymph nodes. J. Exp. Med. 209, 653-660 (2012).

20. Villani, A. C. et al. Single-cell RNA-seq reveals new types of human blood dendritic cells, monocytes, and progenitors. Science 356, pii: eaah4573(2017).

21. Barker, J. E. Sl/Sld hematopoietic progenitors are deficient in situ. Exp. Hematol. 22, 174-177 (1994).

22. Barker, J. E. Early transplantation to a normal microenvironment prevents the development of Steel hematopoietic stem cell defects. Exp. Hematol. 25, 542-547 (1997).

23. Greenbaum, A. et al. CXCL12 in early mesenchymal progenitors is required for haematopoietic stem-cell maintenance. Nature 495, 227-230 (2013).

24. Dar, A. et al. Chemokine receptor CXCR4-dependent internalization and resecretion of functional chemokine SDF-1 by bone marrow endothelial and stromal cells. Nat. Immunol. 6, 1038-1046 (2005).

25. Qian, H. et al. Critical role of thrombopoietin in maintaining adult quiescent hematopoietic stem cells. Cell Stem Cell 1, 671-684 (2007).

26. Yoshihara, H. et al. Thrombopoietin/MPL signaling regulates hematopoietic stem cell quiescence and interaction with the osteoblastic niche. Cell Stem Cell 1, 685-697 (2007)

27. Mendelson, A. \& Frenette, P. S. Hematopoietic stem cell niche maintenance during homeostasis and regeneration. Nat. Med. 20, 833-846 (2014).

28. Guermonprez, P. et al. Inflammatory Flt3l is essential to mobilize dendritic cells and for T cell responses during Plasmodium infection. Nat. Med. 19, 730-738 (2013).

29. Saito, Y., Boddupalli, C. S., Borsotti, C. \& Manz, M. G. Dendritic cell homeostasis is maintained by nonhematopoietic and T-cell-produced Flt3ligand in steady state and during immune responses. Eur. J. Immunol. 43, 1651-1658 (2013).

30. Eidenschenk, C. et al. Flt3 permits survival during infection by rendering dendritic cells competent to activate NK cells. Proc. Natl Acad. Sci. USA 107, 9759-9764 (2010).

31. Ginhoux, F. et al. The origin and development of nonlymphoid tissue CD103 + DCs. J. Exp. Med. 206, 3115-3130 (2009).

32. Malhotra, D. et al. Transcriptional profiling of stroma from inflamed and resting lymph nodes defines immunological hallmarks. Nat. Immunol. 13, 499-510 (2012).

33. Waskow, C. et al. The receptor tyrosine kinase Flt3 is required for dendritic cell development in peripheral lymphoid tissues. Nat. Immunol. 9, 676-683 (2008)

34. Liu, K. et al. Origin of dendritic cells in peripheral lymphoid organs of mice. Nat. Immunol. 8, 578-583 (2007).

35. Caux, C., Dezutter-Dambuyant, C., Schmitt, D. \& Banchereau, J. GM-CSF and TNF-alpha cooperate in the generation of dendritic Langerhans cells. Nature 360, 258-261 (1992).

36. Sallusto, F. \& Lanzavecchia, A. Efficient presentation of soluble antigen by cultured human dendritic cells is maintained by granulocyte/macrophage colony-stimulating factor plus interleukin 4 and downregulated by tumor necrosis factor alpha. J. Exp. Med. 179, 1109-1118 (1994).

37. Proietto, A. I., Mittag, D., Roberts, A. W., Sprigg, N. \& Wu, L. The equivalents of human blood and spleen dendritic cell subtypes can be generated in vitro from human CD34(+) stem cells in the presence of fms-like tyrosine kinase 3 ligand and thrombopoietin. Cell. Mol. Immunol. 9, 446-454 (2012).

38. Balan, S. et al. Human XCR1+ dendritic cells derived in vitro from CD34+ progenitors closely resemble blood dendritic cells, including their adjuvant responsiveness, contrary to monocyte-derived dendritic cells. J. Immunol. 193, $1622-1635$ (2014).

39. Poulin, L. F. et al. Characterization of human DNGR-1+ BDCA3+ leukocytes as putative equivalents of mouse CD8alpha+ dendritic cells. J. Exp. Med. 207, 1261-1271 (2010).

40. Lyman, S. D. et al. Molecular cloning of a ligand for the flt3/flk-2 tyrosine kinase receptor: a proliferative factor for primitive hematopoietic cells. Cell 75, 1157-1167 (1993).

41. Rosnet, O. et al. Human FLT3/FLK2 receptor tyrosine kinase is expressed at the surface of normal and malignant hematopoietic cells. Leukemia 10, 238-248 (1996).

42. McKenna, H. J. et al. Mice lacking flt3 ligand have deficient hematopoiesis affecting hematopoietic progenitor cells, dendritic cells, and natural killer cells. Blood 95, 3489-3497 (2000).

43. Maraskovsky, E. et al. Dramatic increase in the numbers of functionally mature dendritic cells in Flt3 ligand-treated mice: multiple dendritic cell subpopulations identified. J. Exp. Med. 184, 1953-1962 (1996).

44. Pulendran, B. et al. Flt3-ligand and granulocyte colony-stimulating factor mobilize distinct human dendritic cell subsets in vivo. J. Immunol. 165, 566-572 (2000).

45. Onai, N., Obata-Onai, A., Tussiwand, R., Lanzavecchia, A. \& Manz, M. G. Activation of the Flt3 signal transduction cascade rescues and enhances type I interferon-producing and dendritic cell development. J. Exp. Med. 203, 227-238 (2006)

46. Kirkling, M. E. et al. Notch signaling facilitates in vitro generation of crosspresenting classical dendritic cells. Cell Rep. 23, 3658-3672 e3656 (2018).

47. Balan, S. et al. Large-scale human dendritic cell differentiation revealing Notch-dependent lineage bifurcation and heterogeneity. Cell Rep. 24, 1902-1915 el906 (2018).

48. Ding, Y. et al. FLT3-ligand treatment of humanized mice results in the generation of large numbers of $\mathrm{CD} 141+$ and $\mathrm{CD} 1 \mathrm{c}+$ dendritic cells in vivo. J. Immunol. 192, 1982-1989 (2014).

49. Li, Y. et al. A novel Flt3-deficient HIS mouse model with selective enhancement of human DC development. Eur. J. Immunol. 46, 1291-1299 (2016).

50. Yu, C. I. et al. Human CD1c+ dendritic cells drive the differentiation of CD103+ CD8+ mucosal effector T cells via the cytokine TGF-beta. Immunity 38, 818-830 (2013).

51. Itoh, K. et al. Reproducible establishment of hemopoietic supportive stromal cell lines from murine bone marrow. Exp. Hematol. 17, 145-153 (1989).

52. Nakano, T., Kodama, H. \& Honjo, T. Generation of lymphohematopoietic cells from embryonic stem cells in culture. Science 265, 1098-1101 (1994).

53. Ding, L., Saunders, T. L., Enikolopov, G. \& Morrison, S. J. Endothelial and perivascular cells maintain haematopoietic stem cells. Nature 481, 457-462 (2012).

54. Caux, C. et al. CD34+ hematopoietic progenitors from human cord blood differentiate along two independent dendritic cell pathways in response to GM-CSF+TNF alpha. J. Exp. Med. 184, 695-706 (1996).

55. Alcantara-Hernandez, M. et al. High-dimensional phenotypic mapping of human dendritic cells reveals interindividual variation and tissue specialization. Immunity 47, 1037-1050 e1036 (2017).

56. Xu, Y., Zhan, Y., Lew, A. M., Naik, S. H. \& Kershaw, M. H. Differential development of murine dendritic cells by GM-CSF versus Flt3 ligand has implications for inflammation and trafficking. J. Immunol. 179, 7577-7584 (2007).

57. Naik, S. H. et al. Cutting edge: generation of splenic CD8+ and CD8dendritic cell equivalents in Fms-like tyrosine kinase 3 ligand bone marrow cultures. J. Immunol. 174, 6592-6597 (2005).

58. Subramanian, A. et al. Gene set enrichment analysis: a knowledge-based approach for interpreting genome-wide expression profiles. Proc. Natl Acad. Sci. USA 102, 15545-15550 (2005).

59. Spinelli, L., Carpentier, S., Montanana Sanchis, F., Dalod, M. \& Vu Manh, T P. BubbleGUM: automatic extraction of phenotype molecular signatures and comprehensive visualization of multiple Gene Set Enrichment Analyses. BMC Genomics 16, 814 (2015).

60. McGovern, N. et al. Human dermal CD14(+) cells are a transient population of monocyte-derived macrophages. Immunity 41, 465-477 (2014).

61. Becht, E. et al. Evaluation of UMAP as an alternative to t-SNE for single-cell data. bioRxiv https://doi.org/10.1101/298430 (2018).

62. Yin, X. et al. Human blood CD1c+ dendritic cells encompass CD5high and CD5low subsets that differ significantly in phenotype, gene expression, and functions. J. Immunol. 198, 1553-1564 (2017).

63. Dutertre, C. A. et al. Single-cell analysis of human mononuclear phagocytes reveals subset-defining markers and identifies circulating inflammatory dendritic cells. Immunity 51, 573-589 e578 (2019).

64. Bonifaz, L. et al. Efficient targeting of protein antigen to the dendritic cell receptor DEC-205 in the steady state leads to antigen presentation on major histocompatibility complex class I products and peripheral CD8+ T cell tolerance. J. Exp. Med. 196, 1627-1638 (2002).

65. Toksoz, D. et al. Support of human hematopoiesis in long-term bone marrow cultures by murine stromal cells selectively expressing the membrane-bound and secreted forms of the human homolog of the steel gene product, stem cell factor. Proc. Natl Acad. Sci. USA 89, 7350-7354 (1992).

66. Takagi, S. et al. Membrane-bound human SCF/KL promotes in vivo human hematopoietic engraftment and myeloid differentiation. Blood 119, 2768-2777 (2012).

67. Kong, X. F. et al. Disruption of an antimycobacterial circuit between dendritic and helper T cells in human SPPL2a deficiency. Nat. Immunol. 19, 973-985 (2018).

68. Rongvaux, A. et al. Development and function of human innate immune cells in a humanized mouse model. Nat. Biotechnol. 32, 364-372 (2014).

69. Nicolini, F. E., Cashman, J. D., Hogge, D. E., Humphries, R. K. \& Eaves, C. J. NOD/SCID mice engineered to express human $\mathrm{IL}^{-} 3$, GM-CSF and Steel factor constitutively mobilize engrafted human progenitors and compromise human stem cell regeneration. Leukemia 18, 341-347 (2004).

70. Ito, R. et al. Establishment of a human allergy model using human IL-3/GMCSF-transgenic NOG mice. J. Immunol. 191, 2890-2899 (2013).

71. Willinger, T. et al. Human IL-3/GM-CSF knock-in mice support human alveolar macrophage development and human immune responses in the lung. Proc. Natl Acad. Sci. USA 108, 2390-2395 (2011). 
72. Orimo, A. et al. Stromal fibroblasts present in invasive human breast carcinomas promote tumor growth and angiogenesis through elevated SDF-1/ CXCL12 secretion. Cell 121, 335-348 (2005).

73. Dobin, A. et al. STAR: ultrafast universal RNA-seq aligner. Bioinformatics 29, 15-21 (2013)

74. Love, M. I., Huber, W. \& Anders, S. Moderated estimation of fold change and dispersion for RNA-seq data with DESeq2. Genome Biol. 15, 550 (2014).

75. Benjamini, Y. Controlling the false discovery rate: a practical and powerful approach to multiple testing. J. R. Stat. Soc. B Methodol. 57, 289-300 (1995).

76. Wickham, H. ggplot2: elegant graphics for data analysis (Springer Science \& Business Media, 2009)

77. Kamburov, A., Wierling, C., Lehrach, H. \& Herwig, R. ConsensusPathDB-a database for integrating human functional interaction networks. Nucleic Acids Res. 37, D623-628 (2009).

78. Becher, B. et al. High-dimensional analysis of the murine myeloid cell system. Nat. Immunol. 15, 1181-1189 (2014).

79. Finck, R. et al. Normalization of mass cytometry data with bead standards. Cytom. A 83, 483-494 (2013).

80. Newell, E. W., Sigal, N., Bendall, S. C., Nolan, G. P. \& Davis, M. M. Cytometry by time-of-flight shows combinatorial cytokine expression and virus-specific cell niches within a continuum of CD8+ T cell phenotypes. Immunity 36, 142-152 (2012).

81. Parks, D. R., Roederer, M. \& Moore, W. A. A new "Logicle" display method avoids deceptive effects of logarithmic scaling for low signals and compensated data. Cytom. A 69, 541-551 (2006).

82. McInnes, L. \& Healy, J. UMAP: uniform manifold approximation and projection for dimension reduction. Preprint at arXiv:1802.03426 (2018).

\section{Acknowledgements}

P.G. is a CNRS investigator. The research was supported by the NC3Rs, BBSRC, CRUK Rosetree Trust, and King's Health Partners. Authors are recipient of awards and grants BBSRC-BB/M029735/1, CRUK-CIPA C57672/A22369, WWCR-18-0422, ANR-18-IDEX0001, and ANR-17-CE11-0001-01. This work was supported by the Institut Curie, Institut National de la Santé et de la Recherche Médicale, Labex DCBIOL (ANR-10-IDEX-0001-02 PSL and ANR-11-LABX0043), SIRIC INCa-DGOS-Inserm_12554, and ANR JCJC (ANR17-CE15-0004). We thank Michael Ridley, Emily Hanton, and Beth Ormrod for technical assistance and Matthew Nicholls for schematic design. We also want to thank Anthony Nolan Cord Blood Bank and Cell Therapy Centre for providing the cord blood units used in this study. All flow cytometry work was performed within the NIHR Biomedical Research Centre based at Guy's and St. Thomas' NHS Foundation Trust and King's College London.

\section{Author contributions}

G.A., P.B., and O.H. performed the experiments. K.V. and Y.M.K. performed bioinformatic analysis of RNA-seq data. C.A.D. and E.N. performed CyTOF experiments and analysis. K.W. and A.S. performed RNA library preparation and sequencing. J.H. provided reagents and expertise for in vitro cultures. F.G. provided reagents and expertise for CyTOF analysis. G.A. and P.G. designed the experiments and wrote the manuscript. P.G. conceived and supervised the project.

\section{Competing interests}

The authors declare no competing interests.

\section{Additional information}

Supplementary information is available for this paper at https://doi.org/10.1038/s41467020-15937-y.

Correspondence and requests for materials should be addressed to P.G.

Peer review information Nature Communications thanks the anonymous reviewer(s) for their contribution to the peer review of this work.

Reprints and permission information is available at http://www.nature.com/reprints

Publisher's note Springer Nature remains neutral with regard to jurisdictional claims in published maps and institutional affiliations.

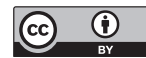

Open Access This article is licensed under a Creative Commons Attribution 4.0 International License, which permits use, sharing, adaptation, distribution and reproduction in any medium or format, as long as you give appropriate credit to the original author(s) and the source, provide a link to the Creative Commons license, and indicate if changes were made. The images or other third party material in this article are included in the article's Creative Commons license, unless indicated otherwise in a credit line to the material. If material is not included in the article's Creative Commons license and your intended use is not permitted by statutory regulation or exceeds the permitted use, you will need to obtain permission directly from the copyright holder. To view a copy of this license, visit http://creativecommons.org/ licenses/by/4.0/.

(C) The Author(s) 2020 